\title{
Modeling the Metabolic Reductions of a Passive Back-Support Exoskeleton
}

Journal of Applied Physiology 2022.

Mohammad Mehdi Alemi ${ }^{1,2,3}$, Athulya A. Simon ${ }^{3}$, Jack Geissinger ${ }^{4}$, and Alan T. Asbeck ${ }^{3, *}$

${ }^{1}$ Center for Advanced Orthopaedic Studies, Beth Israel Deaconess Medical Center, 330 Brookline Ave., Boston 02215, MA, United States of America

${ }^{2}$ Department of Orthopaedic Surgery, Harvard Medical School, Boston 02115, MA, United States of America

${ }^{3}$ Department of Mechanical Engineering, Virginia Polytechnic Institute and State University

Blacksburg, VA, 24061, United States of America

${ }^{4}$ Department of Computer Engineering, Virginia Polytechnic Institute and State University

Blacksburg, VA, 24061, United States of America

*Corresponding author: Alan T. Asbeck (aasbeck@vt.edu)

Corresponding author address: 231 Goodwin Hall, 635 Prices Fork Road, Blacksburg, VA 24061

Keywords: back-support exoskeleton; lifting exoskeleton; energy expenditure; metabolic cost reduction; oxygen consumption; energy modeling

Running head: Modeling Metabolic Reductions of a Back-Support Exoskeleton

\begin{abstract}
Despite several attempts to quantify the metabolic savings resulting from the use of passive backsupport exoskeletons (BSEs), no study has modeled the metabolic change while wearing an exoskeleton during lifting. The objectives of this study were to: 1) quantify the metabolic reductions due to the VTLowe's exoskeleton during lifting; and 2) provide a comprehensive model to estimate the metabolic reductions from using a passive BSE. In this study, 15 healthy adults $(13 \mathrm{M}, 2 \mathrm{~F})$ of ages 20 to 34 years (mean=25.33, $\mathrm{SD}=4.43$ ) performed repeated freestyle lifting and lowering of an empty box and a box with $20 \%$ of their bodyweight. Oxygen consumption and metabolic expenditure data were collected. A model for metabolic expenditure was developed and fitted with the experimental data of two prior studies and the without-exoskeleton experimental results. The metabolic cost model was then modified to reflect the effect of the exoskeleton. The experimental results revealed that VT-Lowe's exoskeleton significantly lowered the oxygen consumption by $\sim 9 \%$ for an empty box and $8 \%$ for a $20 \%$ bodyweight box, which corresponds to a net metabolic cost reduction of $\sim 12 \%$ and $\sim 9 \%$, respectively. The mean metabolic difference (i.e., without-exo minus with-exo) and the $95 \%$ confidence interval were 0.36 and $(0.2-0.52)$ [Watts $/ \mathrm{kg}$ ] for $0 \%$ bodyweight, and 0.43 and $(0.18-0.69)$ [Watts $/ \mathrm{kg}$ ] for $20 \%$ bodyweight. Our modeling predictions for with-exoskeleton conditions were precise, with absolute freestyle prediction errors of $<2.1 \%$. The model developed in this study can be modified based on different study designs, and can assist researchers in enhancing designs of future lifting exoskeletons.
\end{abstract}




\section{New and Noteworthy}

We present a new model of the metabolic cost of repetitive lifting, and how that is affected by wearing a passive back support exoskeleton. We compute the effective biomechanical efficiencies of moving the body and a carried load during lifting, and determine the effect of an exoskeleton's efficiency on its metabolic reduction. This model is useful for understanding the effects of exoskeletons on the body and for designing future exoskeletons.

\section{Introduction}

Lower back pain due to the lifting and lowering overexertion is one of the major sources of injuries and illnesses especially for manual material handling workers. Lower back pain is recognized as the leading cause of activity limitation, disability, and work absence, accounting for $\sim 40 \%$ of lost workdays with estimated direct healthcare expenditure of $\$ 50-\$ 90$ billion annually in the United States $(40,84)$. Earlier epidemiological studies have shown that there is a strong association between the development of lower back pain and repetitive lifting/lowering, bending, and prolonged non-neutral static postures $(20,39,65)$. Different interventions, including workplace modifications $(34,53,58)$, work method training $(27,35,75)$, exercise and education $(13,14,76)$, worker training $(18,21)$, and workspace or tools redesign $(55,71)$ have been proposed as ergonomic solutions to prevent lower back pain.

In recent years, passive back-support exoskeletons (BSEs) have also been proposed as a promising intervention to reduce the risk of low back injuries by offloading the weight of the wearer's torso during various activities such as lifting (e.g., $(2,6,7,56))$, bending/static holding (e.g., $(12,16,50)$ ), and drilling (e.g., $(48,49))$ tasks. An exoskeleton is defined as a wearable, external structure that augments or assists the performance or strength of the user by providing assistive joint moments and/or structural supports (15, 83). Although we and others have reported the benefits of passive BSEs on muscle activity (back and leg muscles) during lifting, assembly, and static holding tasks $(2,3,6,7,16,28,37,56,57,78,80)$, relatively little information is available regarding the efficacy of BSEs in reducing the metabolic costs of lifting.

Several previous works have already calculated or modeled the metabolic cost of lifting when a person is not wearing an exoskeleton (e.g., $(8,23,30,32,33,36,41,51,73,81))$. In these studies, several factors have been reported to affect the lifting metabolic expenditure to various degrees: these include body weight, external load weight, frequency or lifting speed, lifting posture and technique, vertical/horizontal movement of body and external load, gender, physical fitness, environmental conditions (temperature and humidity), and task time duration $(4,8,31,32,36)$. However, based on the physics of lifting activity and/or the experimental designs, the effects of some of these factors are much more significant than others. Most of the previous studies focused more exclusively on the effects of lifting techniques, lifting frequency and external load on metabolic expenditure of lifting. For example, Garg et al. developed a new approach (based on regression equations) to predict the metabolic cost for manual material handling jobs (32). They assumed that a certain activity can be decomposed to several task elements and subsequently, the total metabolic cost of the job can be defined as sum of tasks' energy demands and the energy required for maintaining the body's posture. They validated their model through 48 different jobs and they reported a high correlation coefficient of 0.95 between the predicted and measured metabolic expenditures. To validate their lifting equations, Garg and colleagues (32) compared 
their model with the measured metabolic cost of lifting reported in (8) and (73). Although the estimated results of Garg's model over-predicted Aquilano's model and under-predicted Snook's measured metabolic cost results, the average absolute differences were $\sim 13.2 \%$ and $\sim 6.8 \%$, respectively. Hence, their model provided reasonable prediction accuracy for lifting metabolic cost. Another excellent review of the metabolic cost of lifting is available in (41), where the metabolic cost of stoop and squat lifting was measured at various frequencies (10 and 20 lifts/min) and lifted masses $(1,8.5$ and $17 \mathrm{~kg}$ ) for 10 male professional forestry workers. Welbergen et al. also conducted a study to explore the effect of lifting technique (stoop and squat) and frequency (10 and $20 \mathrm{lifts} / \mathrm{min}$ ) on metabolic cost for a repetitive lifting of a barbell $(19 \mathrm{~kg})$ on 9 male powerlifters (81). Welbergen et al. and Hagen et al. both reported that in a given lifting frequency, the squat lifting had higher metabolic cost and oxygen consumption compared to the stoop lifting. In addition, as expected, for a given lifting technique, higher lifting frequency yielded a higher metabolic cost.

In the past, research studies have confirmed that the increased metabolic cost during lifting is associated with fatigue (decreased capacity for exertion) and has adverse effects on lifting kinematics, lifting power, and postural stability $(47,59,70,74)$. Use of passive BSEs could be an alternative preventive approach to reduce the metabolic cost of lifting. To date, all of the metabolic cost studies with passive BSEs have only focused on quantifying the metabolic cost reductions during repetitive lifting tasks $(5,7,10,11,56,82)$. However, no study has attempted to model the metabolic cost of lifting with an exoskeleton. In this paper, we addressed two questions: 1) is there any significant change in metabolic expenditure during lifting with the VT-Lowe's exoskeleton; and 2) what is a comprehensive model to predict the metabolic cost reduction resulting from using an exoskeleton? The main hypothesis of this research is that wearing the VT-Lowe's exoskeleton would reduce the metabolic cost of lifting since it partially offsets the body weight (specifically torso weight) during lifting. Findings of this study will improve our understanding of the factors that significantly contribute to the metabolic cost of an exoskeleton and will increase the design accuracy and efficiency of future lifting exoskeletons.

\section{Methods and Methods}

\section{Subjects}

A convenience sample of 15 subjects (13 males and 2 females) from university students and local communities was recruited for this study. Their mean (SD) ages, heights and body masses were 25.33 (4.43) years, 174.3 (6.99) $\mathrm{cm}$ and 79.27 (8.77) $\mathrm{kg}$, respectively. The sample size of this study is slightly larger than other comparable studies in which significant differences in metabolic cost were obtained using ten (11) and eleven (10) healthy subjects. The current study was approved by the institutional review board (IRB) of Virginia Tech and written consent forms were obtained from all subjects at the study session. None of the subjects reported any history of musculoskeletal disorders or low back pain in the past 12 months. Further, all of them were capable of lifting at least $25 \%$ of their body weight. 


\section{Preparation}

The overall experimental protocols were initially explained to each subject. In addition, during a 30-minute training procedure, all subjects were asked to wear the exoskeleton to ensure their familiarity with the device. The exoskeleton was adjusted to each subject while they lifted a box weighted with $0 \%$ and $20 \%$ of their bodyweight (BW) in the sagittal plane. They repeated this task until they felt comfortable with the exoskeleton. This familiarization process was performed to minimize the training effects during the experimental testing. In parallel, subjects were given information about the portable calorimeter CosMed K4B2, a system that reliably calculates the metabolic cost of a task by measuring the oxygen uptake and exhaled carbon dioxide $(24,43,61,63)$. Following the training, each subject was given a break of approximately 15 to 20 minutes before the start of the experiment.

\section{Experimental procedures}

In this experiment, a full lifting cycle was defined as (1) bending down to grasp a $0.43 \times 0.31 \times 0.2$ $\mathrm{m}^{3}$ plastic box; (2) lifting the box up from the floor to knuckle height (arms straight); (3) lowering the box back down to the floor; and (4) rising again to the original standing posture. During the experiment, participants performed 6 full lifting cycles per minute using a freestyle form. Note that this is equivalent to the participants performing 6 lifts (bend down and stand up again) with no load, and 6 more lifts while holding the box, per minute. The independent variables were: (1) box weight ( $0 \%$ and $20 \%$ of BW), and (2) exoskeleton interventions (i.e., with and without-exoskeleton). The exoskeleton used was the VTLowe's exoskeleton, as previously described in (6) and (19). The exoskeleton has a mass of $4.5 \mathrm{~kg}$, which was $5.7 \%$ of the mean body mass for the participants in this experiment. Photos of the exoskeleton used in the experiment and a participant during the experiment are shown in Figure 1. The test subjects completed a twelve-minute A-B-A protocol, where they did one condition (e.g., lifting without the exoskeleton) first ("A" step), then did a different condition (e.g., lifting with the exoskeleton) next ("B" step), and then repeated the A step at the end. This procedure ensures that the difference between the A condition and B condition was maintained over time, and the subject did not fatigue. If the subject felt fatigued, the second "A" test would show a higher metabolic rate than the first "A" test. 

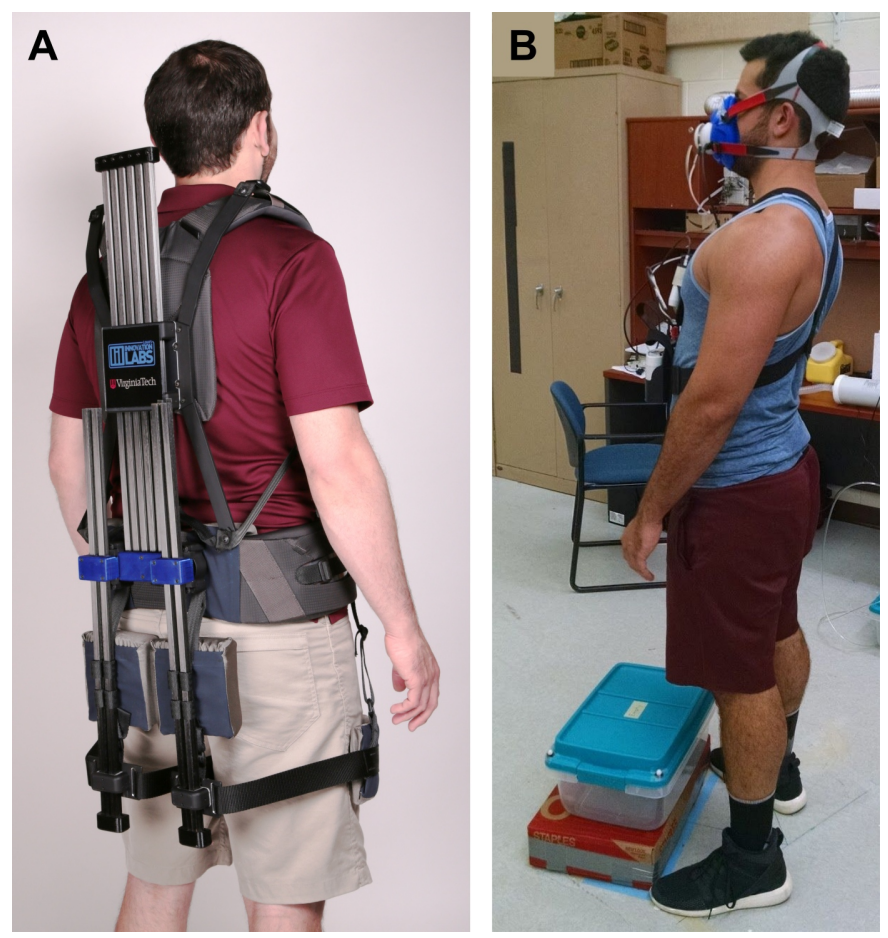

Figure 1: A, photo of the exoskeleton used in the experiment. B, photo of a participant in the experiment completing the without-exoskeleton condition. The box rested on a short platform, and participants could position their feet as they wished. For the $20 \%$ BW conditions, mass was placed inside the box.

During the experimental sessions, to keep the lifting time and speed the same, a narrative script was built using Matlab 9.0 (Mathworks, Natick, MA) and the subjects lifted and returned the box as narrator commanded. Each subject completed the experiment for both $0 \%$ and $20 \%$ of BW in the A-B-A protocol described above. The subjects jogged in place initially for 30 seconds to raise the metabolic rate quickly from the standing baseline. Then, they performed lifting for five minutes, with one complete lifting cycle every ten seconds. Then the exoskeleton is put on (or taken off) within 30 seconds based on the starting "A" step. Next, the subjects continued lifting for three minutes at the same rate and again the exoskeleton is taken off (or put on) and the lifting continued for 3 more minutes. Therefore, for a given box weight, the experimental session took about 12 minutes. To minimize the confounding errors, the presentation order of independent factors (exoskeleton intervention and box weight) were fully randomized during the experiment. Oxygen consumption was collected during the entire session for each box weight. Enough rest (15-20 minutes) was provided to subjects after finishing each session to ensure that the heart rate and muscle conditions went back to resting conditions.

\section{Metabolic power calculation and statistical analysis}

The experimental data from metabolic cost system was initially processed using Matlab. The consumed oxygen and carbon dioxide produced were filtered using a $4^{\text {th }}$ order Butterworth filter with cutoff frequency of $0.33 \mathrm{~Hz}$. It should be noted that it takes several minutes for the metabolic rate to stabilize. The initial subject's jogging in place raised the metabolic rate rapidly to be close to that of steady-state lifting, shortening the time to reach a steady-state metabolic rate. After the subject jogged, an additional stabilization period was provided to ensure the metabolic rate reflected that of steady-state lifting. 
Therefore, based on the obtained results, the first 3 minutes of the metabolic cost data were dropped from analysis. In each step (i.e., $\mathrm{A}$ and $\mathrm{B}$ ), the $\mathrm{O} 2$ consumption and produced $\mathrm{CO} 2$ were averaged across time for the last 1.5 minutes of that step interval to produce a single value for each participant. For this study, the metabolic power $M$ was calculated using the Brockway equation excluding the Nitrogen term (17): $M=16.58 \dot{V} O_{2}+4.51 \dot{V} C O_{2}$, where $\dot{V} O_{2}$ and $\dot{V} C O_{2}$ are in [L/sec] and $M$ is in [Watts].

In this study, all statistical analyses were performed by SPSS Statistics 26 (IBM SPSS Statistics, USA). A paired t-test was used to compare the normalized metabolic cost [Watts $/ \mathrm{kg}$ ] and oxygen consumption [L/min] of the first and third steps for all subjects. This first analysis examines whether or not the subjects fatigued during each experimental session. Additionally, a two-way repeated-measures analyses of variance (ANOVA) was performed to examine the effect of box weights (i.e., 0\% and 20\%) and exoskeleton intervention on metabolic cost and oxygen consumption. A post hoc paired t-test was subsequently performed on the ANOVA results and the mean differences (i.e., without-exo minus withexo) and their corresponding 95\% confidence intervals and p-values were reported for both box weights (Table 1). In order to investigate the effect of exoskeleton on metabolic cost reduction, the metabolic rate in first and third steps (first "A" and second "A") were averaged and then statistically compared with the second step (step "B"). Partial eta-squared $\left(\eta_{P}{ }^{2}\right)$ as a measure of effect sizes was also provided for statistically significant effects of ANOVA findings. For all statistical analyses, the alpha level of 0.05 was set as the statistical level of significance.

\section{Modeling Metabolic Cost Reductions}

In the following sections, we explore the general question of, what metabolic reduction should we expect from an exoskeleton? To understand this, we first present a theoretical model for metabolic cost prediction during lifting without an exoskeleton, and fit the model with the experimental results of two other studies that conducted stoop and squat lifting without an exoskeleton $(41,81)$ (Part A), as a baseline for comparison. Next, the model was modified and fitted with the measured results of the withoutexoskeleton conditions from our current study (Parts B and C). In both Parts A and C, we fit experimental data to the model to calculate the effective biomechanical efficiencies of the body during lifting without an exoskeleton. Next, the developed model for without-exoskeleton conditions was again modified and some new terms were added to it to reflect the contributions of an exoskeleton (Part D). We then use the model to predict the metabolic reduction in the present study, and compare it to our experimental results (Part E).

\section{Part A: Developing a prediction model for the metabolic cost of lifting}

We first consider modeling the metabolic cost of lifting a box without an exoskeleton. This question has been addressed by several previous researchers, including (32), (69), (23), and (81); our modeling framework improves upon their results by creating a physics-based and intuitive set of equations. Contrary to the previous studies mentioned, our modeling framework decomposes the biomechanical efficiencies of the box and body to include both the lowering and lifting phases, which is important to understand to accurately model different activities. Our model can be easily modified to predict the lifting metabolic cost of different lifting scenarios to as long as the corresponding lifting kinematics and anthropometric data are available.

To predict the metabolic cost of a certain lifting activity, the entire lifting cycle is divided into several task elements and the metabolic cost of lifting is modeled for each task element, as proposed by (32). Then, the total metabolic cost of the cycle is calculated using equation (1) in (32), in which the 
different elements are added together and scaled by their respective times. In the case of repetitive lifting, there are contributions from lifting the body and a held mass, lowering the body and a held mass, and a baseline metabolic cost for standing upright. We assume that the lifters are continuously holding a mass during the lowering and lifting phases. They lower it down (so as to place the mass on the ground, although in this model they do not release it), then stand up again (with the mass), at which point they remain standing until the start of the next cycle. As specified in (69), this requires a mechanical power $P$ to raise the body and box:

$$
P=\left(\frac{L}{60}\right)\left(\left|W_{b o x}\right|+\left|W_{B o d y}\right|\right) \quad \text { (Equation 1a) }
$$

where $\left|W_{\text {box }}\right|=m_{B o x} g \Delta h_{B o x}$ and $\left|W_{B o d y}\right|=m_{B o d y} g \Delta h_{B o d y}$ are the mechanical work in Joules required to lift the box and body center of mass (COM), respectively, and lifting occurs at $L$ lifts per minute. The box has mass $m_{B o x}[\mathrm{~kg}]$, the body has mass $m_{B o d y}[\mathrm{~kg}]$, the box and body COM change heights $\Delta h_{B o x}$ and $\Delta h_{B o d y}[\mathrm{~m}]$, respectively, and $g$ is the gravitational constant. Note that this only includes the power to lift the box; lowering the box results in the opposite mechanical power, so the entire lifting cycle has a net mechanical power of zero. Metabolically, there is a cost to both lifting and lowering the body and box masses, as well as a cost for the body to remain alive and standing (i.e., to maintain its posture). The metabolic cost of standing is a constant $(32,33,41)$, which we define as $M_{\text {Standing. }}$ Garg and colleagues reported that the resting metabolic cost for a person with body weight $\mathrm{BW}$ is equal to $0.024 \mathrm{BW}$ [Kcal $/ \mathrm{min}$ ] which is approximately $1.67 \mathrm{BW}$ [Watts] (32). Therefore, for a $75 \mathrm{~kg}$ person, the metabolic cost of standing is about $1.8 \mathrm{kcal} / \mathrm{min} \sim 125.5 \mathrm{Watts}$ (33). Hagen and colleagues also plotted the mean oxygen consumption during standing $(\sim 0.36 \mathrm{~L} / \mathrm{min} \mathrm{O})$ in Figure 1 of their paper (41). Assuming that $1 \mathrm{~L} / \mathrm{min} \mathrm{O} 2 \sim 348.68$ Watts, the metabolic cost of standing for their study with mean body mass of $78.30 \mathrm{~kg}$ is about 125 Watts. In the current study, the metabolic cost of standing was assumed to be 125 Watts for the mean body mass of $78.3 \mathrm{~kg}$ (standing metabolic cost in (41) was set as the reference). Later in this paper, to predict the metabolic cost of standing in other studies that have different mean body masses, the standing metabolic cost was normalized with respect to the corresponding mean body weight in those studies.

To model the metabolic cost of lifting and lowering, we must include the biomechanical efficiency of the body in performing these tasks. Putting the components together results in Equation (1) below:

$$
\begin{gathered}
M_{\text {Lifting,NoExo }}= \\
M_{\text {Standing }}+\left(\frac{L}{60}\right)\left(\left|W_{\text {Box }}\right|\left(K_{\text {Box,lower }}+K_{\text {Box,lift }}\right)+\left|W_{\text {Body }}\right|\left(K_{\text {Body,lower }}+K_{\text {Body,lift }}\right)\right)= \\
M_{\text {Standing }}+\left(\frac{L}{60}\right)\left(K_{\text {Box }}\left|W_{\text {Box }}\right|+K_{\text {Body }}\left|W_{\text {Body }}\right|\right) \quad \text { (Equation 1) }
\end{gathered}
$$

In Equation (1), $M_{\text {Lifting,NoExo }}$ is the average metabolic cost, in Watts, of repetitive lifting at $L$ lifts per minute. The coefficients $K_{B o d y, l o w e r}$ and $K_{B o d y, l i f t}$ are the inverse of the effective biomechanical efficiency to lower and raise the mass of the body during the lowering and lifting phases, respectively. Similarly, $K_{B o x, l o w e r}$ and $K_{B o x, l i f t}$ are the inverse of the effective biomechanical efficiency to lower and raise the box during the lowering and lifting phases, respectively. Furthermore, $K_{B o x}$ (i.e., $K_{B o x}=$ $K_{B o x, l o w e r}+K_{B o x, l i f t}$ ) and $K_{B o d y}$ (i.e., $\left.K_{B o d y}=K_{B o d y, l o w e r}+K_{B o d y, l i f t}\right)$ are the inverses of the effective biomechanical efficiency of box and body during the whole cycle, respectively.

In general, the metabolic cost of lifting depends on the physics and task elements of lifting. Here, to compute the predicted metabolic cost for different lifting types (i.e., stoop, squat, etc.) in Equation (1), 
we need to determine the values of $K_{B o x}$ and $K_{B o d y}$ associated with each lifting type. The other terms in Equation (1) can be calculated from either anthropometric or kinematics data. In order to calculate the values of $K_{B o x}$ and $K_{B o d y}$ for stoop and squat, we performed a least-squares fit of Equation (1) with the experimental data (stoop and squat at $10 \mathrm{lifts} / \mathrm{min}$ ) from (81) and (41) (see Appendix A). While the current study did not include stoop or squat lifts, we compute these values for comparison to the freestyle lifting done in our study.

As explained in the introduction, in both of these studies, the performed repetitive lifting procedure was the same except that while the external weight was $19 \mathrm{~kg}$ in (81), (41) used three external weights (1, 8.5 and $17 \mathrm{~kg}$ ). The lifting procedure of both studies included three phases: 1) initially the subject lowered the external load from knuckle height to the floor; 2) the subject lifted the external load from floor to knuckle height (same posture as at the beginning); and 3) the subject was standing until the next metronome beep to start lowering the external load again. In (81), the change in height of the barbell can be calculated to be $0.66 \mathrm{~m}$ and $0.64 \mathrm{~m}$ for stoop and squat, respectively, from numbers provided in the paper. In the (41) study, the change in box height was approximated to be $0.59 \mathrm{~m}$ as the knuckle height was estimated to be $80 \mathrm{~cm}$ and the vertical displacement between the box handle and the bottom of the box was equal to $21 \mathrm{~cm}$ (see Appendix A). The knuckle height was estimated based on the subject mean height and an equation relating stature and knuckle height derived from data in (38) (see Table C in Appendix A).

Changes in body center of mass (COM) height for (81) were estimated to be $0.32 \mathrm{~m}$ (stoop) and $0.46 \mathrm{~m}$ (squat); and were estimated to be between 0.31-0.33 $\mathrm{m}$ (stoop) and 0.42-0.49 $\mathrm{m}$ (squat) depending on the weight lifted for (41) (details in Appendix A). These values were calculated using the equations in Table D of Appendix A based on the vertical displacement of the box COM and the average body height in each experiment. Since the COM height was not measured in (41) or (81), the change in the COM height was estimated based on the change in height of the mass lifted; equations relating these two quantities were provided in (69), and are duplicated in Table D of Appendix A.

Our model assumes that the lifting is quasi-static, so we just consider the potential energy change when evaluating the metabolic cost. This assumption is reasonable, since the kinetic energy of the body is a relatively small fraction of the total energy during lifting. One study (72) found that the kinetic energy of the body's center of mass was only 3.2-6.1\% of the total energy of the center of mass (stoop lifting corresponded to $3.2 \%$, and squat lifting to $6.1 \%$ ), and similar results were found by (22) and (67).

\section{Part B: Modifying the metabolic cost prediction model to match the current study (without exoskeleton)}

In this section, we use the model from Part A to develop a metabolic cost prediction model for the experimental results of the without-exoskeleton conditions measured in this paper. In our study, a complete lifting cycle includes four distinct phases: 1) lowering the body without box, 2) lifting the body with box, 3) lowering the body with box, and 4) lifting the body without box. After the fourth phase, the subjects were standing without the box until the Matlab script commanded them again to lower their body. Thus, in the current study subjects were lifting and lowering the external load in only half of the lifting and lowering phases, whereas in the experimental designs in (81) and (41), participants held the load constantly. Equation 2 presents the prediction metabolic cost of the whole task (including standing phase) for our experimental design (without-exoskeleton conditions). 


$$
\begin{gathered}
M_{\text {Lifting,NoExo,OurExp }}= \\
M_{\text {Standing }}+\left(\frac{L}{60}\right)\left(K_{\text {Box }}\left|W_{\text {Box }}\right|+K_{\text {Body }}\left|W_{\text {Body }}\right|\right)+\left(\frac{L}{60}\right)\left(K_{\text {Body } y}\left|W_{\text {Body }}\right|\right)= \\
M_{\text {Standing }}+\left(\frac{L}{60}\right)\left(K_{\text {Box }}\left|W_{\text {Box }}\right|+2 K_{\text {Body }}\left|W_{\text {Body }}\right|\right) \quad \text { (Equation 2) }
\end{gathered}
$$

In Equation (2), $M_{\text {Lifting,NoExo,OurExp }}$ is the predicted metabolic cost of one complete lifting cycle (plus standing time waiting for next cycle) and it is calculated in Watts, and the other terms are the same as those in Equation (1).

\section{Part C: Fitting the without-exoskeleton metabolic cost prediction model to our experiment to calculate the effective biomechanical efficiency of box and body during freestyle lifting}

To obtain the effective box and body biomechanical efficiency in metabolic cost prediction of our current study, we fitted the without-exoskeleton prediction model with the corresponding experimental data. It was necessary to fit the data because in the previous studies, stoop and squat lifting styles were used, while in our study freestyle lifting was performed, and the biomechanical efficiencies are different for different lift types. In our study, each complete lifting cycle occurred at 6 cycles/min, so $L=6$ in Equation (2). Moreover, the vertical displacement of the box COM for without-exoskeleton conditions was obtained as $0.55 \mathrm{~m}$ (see Table B in Appendix A). The change in box COM was computed based on two values: 1) the box handle height (vertical distance between box handle and the bottom of the box) that was measured to be $22.5 \mathrm{~cm}$; and 2) the knuckle height, which was calculated as $77.0 \mathrm{~cm}$ by inputting the average body height $(\sim 1.74 \mathrm{~m})$ into the provided equation in Table $\mathrm{C}$ of Appendix A. To find the vertical displacement of body's COM, equations in Table D of Appendix A were used. These equations related the body's COM height to the box height during lifting, with both quantities normalized to the person's height. Substituting a normalized box height change of $0.55 \mathrm{~m} / 1.74 \mathrm{~m}=0.31$ into these equations resulted in the body COM height changes of $0.29 \mathrm{~m}, 0.39 \mathrm{~m}$, and $0.35 \mathrm{~m}$ for stoop, squat and freestyle lifting, respectively, for an empty box, and body COM height changes of $0.30 \mathrm{~m}, 0.45 \mathrm{~m}$, and $0.39 \mathrm{~m}$ for the $20 \%$ BW box. Note that the body COM height changes are slightly larger for a heavy box since people crouch down more to lift heavy loads, as measured by (72). By inserting all of the necessary parameters in the without-exoskeleton metabolic cost model, the model was linearly best fit with our withoutexoskeleton experimental data to calculate the biomechanical efficiency of lifting a box and the body during freestyle lifting. The resulting $K$-values were then used in predicting the metabolic cost of lifting for with-exoskeleton conditions (Part E).

\section{Part D: Developing a prediction model to estimate the metabolic cost of lifting with an exoskeleton}

In this section, the metabolic cost prediction equation (Equation (1)) is modified and some additional terms are added to it to reflect the effect of an exoskeleton on the metabolic cost of lifting. Later, in Part E, the model is matched to the variables in our experiment, and validated with our experimental results of with-exoskeleton conditions. Our model of the metabolic cost of lifting a mass repeatedly (as with Equation (2)) while wearing an exoskeleton is as follows: 


$$
\begin{aligned}
M_{\text {Lifting }, \text { Exo }}= & M_{\text {Standing,Exo }} \\
+ & \left(\frac{L}{60}\right)\left(\left|W_{\text {Box }}\right|\left(K_{\text {Box }, \text { lower }}+K_{\text {Box }, \text { lift }}\right)\right. \\
+ & \left(\left|W_{\text {Body }}\right|+\left|W_{\text {ExoMass }}\right|\right)\left(K_{\text {Body,lower }}+K_{\text {Body }, \text { lift }}\right) \\
- & \left.X\left(K_{\text {Exo,lower }}+\eta_{\text {exo }} K_{\text {Exo,lift }}\right)\right) \\
=M_{\text {Standing }, \text { Exo }}+\left(\frac{L}{60}\right)\left(K_{\text {Box }}\left|W_{\text {Box }}\right|+K_{\text {Body }}\left(\left|W_{\text {Body }}\right|+\left|W_{\text {ExoMass }}\right|\right)-\right. & \left.\quad X\left(K_{\text {Exo,lower }}+\eta_{\text {exo }} K_{\text {Exo,lift }}\right)\right) \quad \text { (Equation 3) }
\end{aligned}
$$

In Equation (3), $M_{\text {Lifting,Exo }}$ and $M_{\text {Standing,Exo }}$ are the predicted metabolic cost of lifting for withexoskeleton conditions [Watts] and the metabolic cost of standing [Watts] while wearing an exoskeleton, respectively. $\left|W_{\text {Exomass }}\right|=m_{E x o} g \Delta h_{E x o}$ is the mechanical work required to change the exoskeleton's center of mass during lifting and lowering [J]. The exoskeleton has mass $m_{E x o}[\mathrm{~kg}]$ and it changes height $\Delta h_{E x o}[\mathrm{~m}] . X$ and $\eta_{\text {exo }}$ are the mechanical work that exoskeleton stores during lowering [J] and the mechanical efficiency of exoskeleton for energy storage and return, respectively: the exoskeleton returns $\eta_{\text {exo }} X$ Joules to the body as the wearer stands. $K_{E x o, l o w e r}$ and $K_{E x o, l i f t}$ are inverse of the effective biomechanical efficiencies of how well the body makes use of the energy stored and returned by the exoskeleton during the lowering and lifting phases, respectively. These include the contributions of all muscles in the body, including the back, stomach, and legs. All other variables are the same as in Equations 1 and 2.

A detailed explanation of the terms in this equation is as follows. First, we consider the metabolic cost of standing while wearing an exoskeleton $\left(M_{\text {Standing,Exo }}\right)$. According to (62), standing with an external load results in a metabolic rate of $M_{\text {Standing,Exo }}=1.5 m_{\text {Body }}+2.0\left(m_{\text {Body }}+m_{\text {Exo }}\right)\left(m_{\text {Exo }} / m_{\text {Body }}\right)^{2}$, where here the external load has been assumed to be equal to the exoskeleton's mass $m_{E x o}$. For a body mass of only $50 \mathrm{~kg}$, an exoskeleton mass of $4.8 \mathrm{~kg}$ is needed to result in a metabolic increase of $1 \mathrm{~W}$; the average body mass of an adult is larger than $50 \mathrm{~kg}$, and thus an exoskeleton mass of $4.8 \mathrm{~kg}$ results in a metabolic increase even smaller than $1 \mathrm{~W}$. Thus, for passive back-support exoskeletons, which usually have masses less than $4.8 \mathrm{~kg}(2,6,19,52,54,77), M_{\text {Standing,Exo }} \cong M_{\text {Standing }}$.

Second, while the mass of the exoskeleton does not contribute much to the standing metabolic rate, it must be considered during lifting and lowering since the body must lift and lower its mass. To include this, we assumed that the mechanical work of body and exoskeleton could be grouped together: the center of mass of a passive back-support exoskeleton is typically located near the wearer's waist, close to the center of mass for a human. Thus, the work to lift the exoskeleton's mass $\left|W_{\text {ExoMass }}\right|$ is multiplied by the body terms $K_{\text {Body,lower }}$ and $K_{\text {Body,lift }}$.

Regarding the energy storage provided by the exoskeleton, there are two additional terms for metabolic cost prediction. First, during bending down while wearing the exoskeleton, the exoskeleton absorbs $X$ Joules of mechanical work, which consequently can reduce the metabolic cost of lowering compared to without-exoskeleton conditions. Note that the energy absorbed by the exoskeleton originally comes from the wearer's potential energy due to their standing upright. This potential energy is normally lost as the person lowers, but the exoskeleton captures and stores some of it, and then the exoskeleton returns it to the person later as they stand upright again. This assumes that the exoskeleton creates a torque smaller than that necessary to support the wearer's torso 
during lifting, i.e., the wearer does not have to fight against the exoskeleton during the lowering phase. This assumption is true for all passive back exoskeletons presented in the literature thus far, as they create peak torques of less than half of the torque required to support the torso (19). The torques created by the exoskeleton and activity of the back and stomach muscles during lifting is discussed in much greater detail in Appendix B. Second, during lifting phase, the exoskeleton returns to the wearer $\eta_{\text {exo }} X$ Joules of mechanical work where $\eta_{\text {exo }}$ is the mechanical efficiency of exoskeleton for energy storage and is less than 1 , as some energy is lost due to friction and hysteresis in the exoskeleton. This value is a mechanical property of the exoskeleton and can be measured experimentally. In general, the stored energy $X$ depends on the bending angle of exoskeleton and if the exoskeleton bends more, more energy is stored; thus, values of $X$ need to be determined separately for stoop, squat, and freestyle lifts with different load heights.

It is also useful to note the expected metabolic power savings due to using an exoskeleton, by subtracting $M_{\text {Lifting,NoExo }}$ (Equation (1)) from $M_{\text {Lifting,Exo }}$ (Equation (3)). This leads to a savings of

$$
\begin{aligned}
\Delta M_{\text {Lifting,Exo }}= & M_{\text {Lifting,Exo }}-M_{\text {Lifting,NoExo }} \\
& =M_{\text {Standing,Exo }}-M_{\text {Standing }} \\
& +\left(\frac{L}{60}\right)\left(K_{\text {Body }}\left|W_{\text {ExoMass }}\right|-X\left(K_{\text {Exo,lower }}+\eta_{\text {exo }} K_{\text {Exo,lift }}\right)\right)
\end{aligned}
$$

(Equation 4)

For most passive exoskeletons, $M_{\text {Standing,Exo }} \cong M_{\text {Standing }}$ so

$$
\Delta M_{\text {Lifting,Exo }}=\left(\frac{L}{60}\right)\left(K_{\text {Body }}\left|W_{\text {ExoMass }}\right|-X\left(K_{\text {Exo,lower }}+\eta_{\text {exo }} K_{\text {Exo,lift }}\right)\right)
$$

(Equation 5)

It is interesting to note that this is only a function of the exoskeleton parameters (mass, energy storage, efficiency), the vertical height that the exoskeleton's mass must be moved during a lift, and the $K$ terms relating to how much energy the body will use in lifting the exoskeleton's mass or save from the exoskeleton's energy storage and return. It is not a function of the mass lifted; thus, we would expect the same metabolic savings (in Watts) for different masses lifted. However, the body's center of mass does move slightly more for heavier load masses (potentially so the lifter can get under the box more), so the prediction will vary a small amount due to that effect.

For our specific experiment, similar to how Equation (1) was modified to reach Equation (2), Equation (3) can be modified to be:

$$
\begin{aligned}
& M_{\text {Lifting,Exo,OurExp }}=M_{\text {Standing,Exo }} \\
& +\left(\frac{L}{60}\right)\left(\left|W_{\text {Box }}\right|\left(K_{\text {Box,lower }}+K_{\text {Box }, \text { lift }}\right)\right. \\
& \left.+2\left(\left|W_{\text {Body }}\right|+\left|W_{\text {Exo }}\right|\right)\left(K_{\text {Body,lower }}+K_{\text {Body,lift }}\right)-2 X\left(K_{\text {Exo,lower }}+\eta_{\text {exo }} K_{\text {Exo,lift }}\right)\right) \\
& =M_{\text {Standing,Exo }}+\left(\frac{L}{60}\right)\left(K_{\text {Box }}\left|W_{\text {Box }}\right|+2 K_{\text {Body }}\left(\left|W_{\text {Body }}\right|+\left|W_{\text {Exo }}\right|\right)-\right. \\
& \left.\quad 2 X\left(K_{\text {Exo,lower }}+\eta_{\text {exo }} K_{\text {Exo,lift }}\right)\right) \quad \text { (Equation 3b) }
\end{aligned}
$$

with $L=6$ as before, and Equation 5 can be modified to be:

$$
\Delta M_{\text {Lifting,Exo }}=\left(\frac{2 L}{60}\right)\left(K_{\text {Body }}\left|W_{\text {ExoMass }}\right|-X\left(K_{\text {Exo,lower }}+\eta_{\text {exo }} K_{\text {Exo,lift }}\right)\right)
$$


(Equation 5b)

\section{Part E: Validating the prediction model by estimating the metabolic cost of lifting with an exoskeleton in our experiment}

We next compute the metabolic cost of lifting in our experiment by substituting values into Equations (3b) and (5b). In our experiment, the values of these variables are as follows. Similar to the without-exoskeleton conditions, the vertical displacement of the box COM for with-exoskeleton conditions was obtained to be $0.55 \mathrm{~m}$ (Table B in Appendix A) and the body's COM height change was found by the equations in Table D in Appendix A. We use $\eta_{\text {exo }}=0.95$ based on measurements of our exoskeleton reported in (19). We use $X=59.3 \mathrm{~J}$ for freestyle lifting, $X=61.8 \mathrm{~J}$ for stoop lifting, and $X=51.5 \mathrm{~J}$ for squat lifting, all of which were measured in (19) for the same exoskeleton and box height as in the current experiment. Also, note that the calculated (best-fit) $K$-values for without-exoskeleton freestyle lifting from Part $\mathrm{C}$ were used in this section to validate our metabolic cost model for with-exoskeleton conditions. The calculated (best-fit) $K$-values for stoop and squat lifting from Part A were additionally used to predict the metabolic cost of stoop and squat lifting in this section, for a general comparison to the results of freestyle lifting.

All of the factors in equations (3) and (3b) are thus known except $K_{\text {Exo,lower }}$ and $K_{\text {Exo,lift }}$. Since the exoskeleton $K$-values $\left(K_{E x o, l o w e r}\right.$ and $\left.K_{E x o, l i f t}\right)$ are unknown, four assumptions about their values were investigated, and the resulting predictions compared to gain insight into the actual values. Specifically, there are two major possibilities that we compare. The first is if the $K$-values for the exoskeleton are equal to $K$-values for the body or the box. The second issue is how to distinguish between the values of $K_{\text {Exo,lower }}$ and $K_{\text {Exo,lift }}$. With our experimental data, it is not possible to distinguish between $K_{\text {Exo,lower }}$ and $K_{\text {Exo,lift }}$ since the exoskeleton is worn continuously. In general, to distinguish between these values, an exoskeleton must be used that would provide assistance torques either during the lift or lower phase but provide zero or much less torque during the other phase. This can be accomplished by either an active exoskeleton or a passive exoskeleton with an adjustable efficiency. Since we cannot distinguish between these values with our exoskeleton, we consider two possibilities for the ratio between the lowering and lifting $K$-values. One option is that they are equal to each other $\left(K_{\text {Exo,lower }}=K_{\text {Exo,lift }}\right)$, and the other is that $K_{\text {Exo,lower }}=0.25 K_{\text {Exo,lift }}$. Since these $K$-values are the inverse of the effective biomechanical efficiency, this corresponds to either lifting requiring the same amount of energy as lowering, or lifting requiring four times as much energy as lowering, respectively. The ratio of 4:1 for lifting to lowering seems to be an upper bound for isolated muscle and whole-body activities, where concentric muscle contraction requires between 2-4 times as much energy as eccentric muscle contraction $(1,22,44,64)$; we use $4: 1$ as a conservative value. Similarly, we use the ratio of 1:1 for lifting to lowering as a lower bound on the possible ratio. If $K_{E x o, l o w e r}=0.25 K_{\text {Exo,lift }}$, the sum of $K_{\text {Exo,lower }}+K_{\text {Exo,lift }}$ is set equal to either $K_{B o d y}$ or $K_{B o x}$.

Thus, we make four assumptions for comparison as follows: in Assumption 1, it is assumed that the $K$-values for the exoskeleton are equal to $K$-values for the body $\left(K_{E x o, l o w e r}=K_{B o d y, l o w e r}, K_{E x o, l i f t}=\right.$ $\left.K_{B o d y, l i f t}\right)$ and $K_{B o d y, l o w e r}=K_{B o d y, l i f t}$. Assumption 2 is similar to Assumption 1 except that $K_{B o d y, l o w e r}$ is considered to be $0.25 K_{\text {Body,lift }}$. In Assumption 3, it is assumed that the exoskeleton $K$-values are equal to box $K$-values $\left(K_{\text {Exo,lower }} \approx K_{\text {Box,lower }}, K_{\text {Exo,lift }} \approx K_{B o x, \text { lift }}\right)$ while $K_{\text {Box,lower }} \approx K_{\text {Box,lift }}$. Finally, Assumption 4 is similar to Assumption 3 except that $K_{B o x, \text { lower }}$ is considered to be $0.25 K_{B o x, l i f t}$. These 
assumptions have been made due to our inability to determine the corresponding box and body $K$-values during lowering and lifting phases separately (i.e., $K_{B o x, l o w e r}, K_{B o x, l i f t}, K_{B o d y, l o w e r}, K_{B o d y, l i f t}$ ).

Note that to calculate the $K_{E x o, l o w e r}$ and $K_{E x o, \text { lift }}$ for freestyle lifting, the best-fit $K_{B o x}$ and $K_{B o d y}$ values from Part $\mathrm{C}$ (i.e., without-exoskeleton conditions in our experiment) were used. For metabolic cost prediction of stoop and squat lifting in with-exoskeleton conditions (as a comparison to freestyle lifting, even though these were not experimentally tested), the best-fit $K$-values from Part A were used for calculation of $K_{\text {Exo,lower }}$ and $K_{\text {Exo,lift }}$.

\section{Results}

\section{Experimental Results}

In our experiment, there were no significant changes in normalized metabolic cost $(0 \% \mathrm{BW}: p=$ $0.980,20 \% \mathrm{BW}: p=0.818)$ and oxygen consumption ( $0 \% \mathrm{BW}: p=0.612,20 \% \mathrm{BW}: p=0.967)$ between the first and third steps. This confirmed that the subjects did not experience fatigue during the experiment. The two-way ANOVA results showed that the box weight and exoskeleton intervention both had significant fixed effects on normalized metabolic cost (box weight $\left(p<0.0001, \eta_{P}{ }^{2}=0.939\right)$, and exo intervention ( $p$ $\left.<0.0001, \eta_{P}{ }^{2}=0.598\right)$ ) and oxygen consumption (box weight $\left(p<0.0001, \eta_{P}{ }^{2}=0.938\right.$ ), and exo intervention $\left.\left(p<0.0001, \eta_{P}{ }^{2}=0.586\right)\right)$. In other words, VT-Lowe's exoskeleton can significantly reduce metabolic cost and oxygen consumption during lifting of both box weights. Moreover, no significant interaction effects were observed between box weights and exoskeleton intervention for normalized metabolic cost $\left(p=0.423, \eta_{P}{ }^{2}=0.046\right)$ and oxygen consumption $\left(p=0.494, \eta_{P}{ }^{2}=0.034\right)$.

Figure 2 depicts the plots of paired participant data for the normalized metabolic expenditure [Watts $/ \mathrm{kg}$ ] and mean oxygen consumption [L/min] for both box weights and exoskeleton intervention. Additionally, Table 1 provides the mean and standard deviation of the normalized metabolic expenditure and mean oxygen consumption, as well as the statistical findings of the paired t-test on the difference between with and without-exoskeleton conditions for both box weights. As shown in Figure 2 and Table 1, for both box weights, the mean normalized metabolic cost and oxygen consumption were reduced while wearing the exoskeleton. 

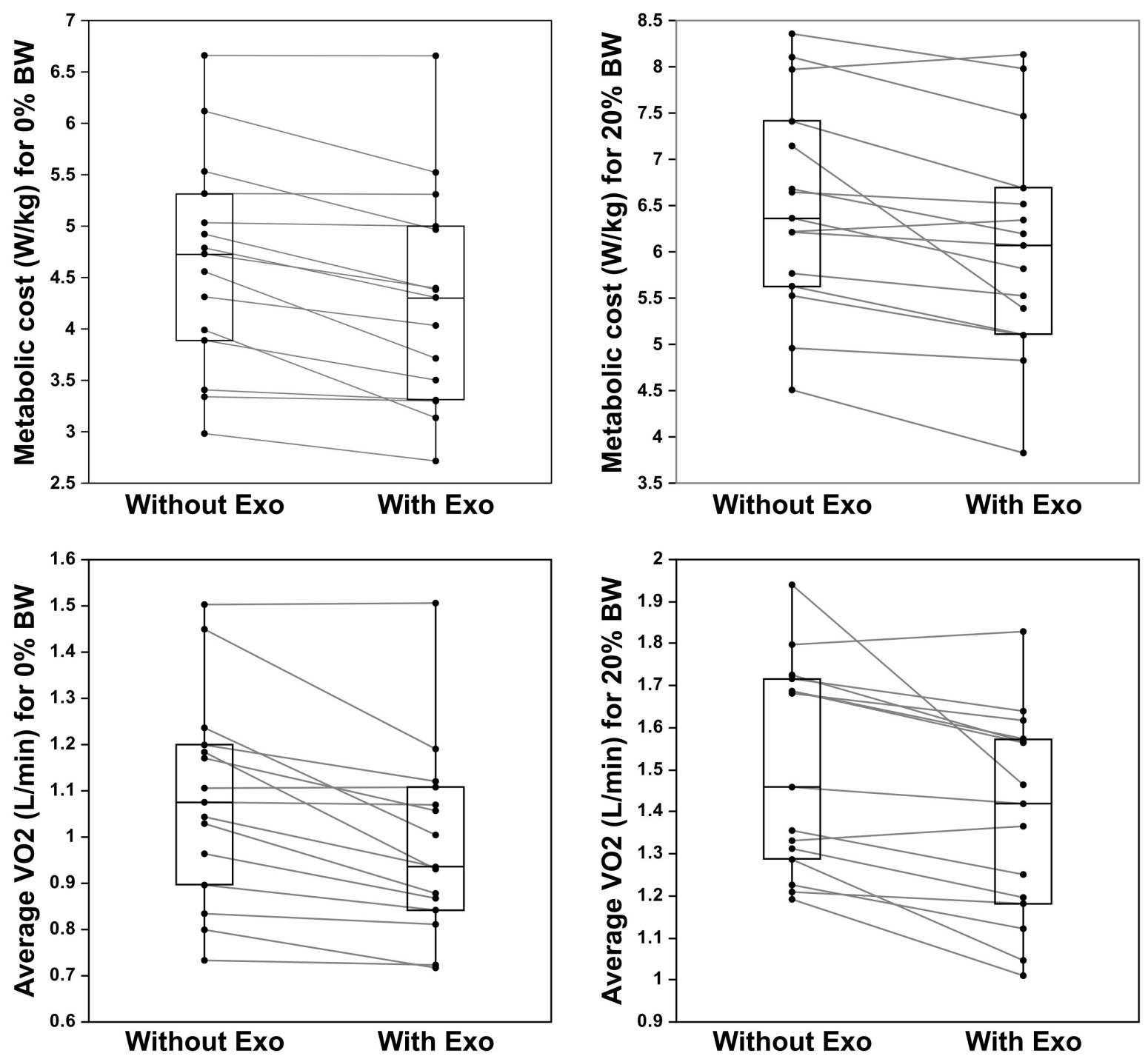

Figure 2: Paired participant data for normalized metabolic expenditure and oxygen consumption, showing the median and interquartile range on each plot $(\mathrm{N}=15 ; 13 \mathrm{M} \& 2 \mathrm{~F})$.

Table 1: The effect of exoskeleton on normalized metabolic cost (Watts $/ \mathrm{kg}$ ) and average V02 consumption (L/min). Note that in the paired $\mathrm{t}$-test analysis $(\mathrm{N}=15 ; 13 \mathrm{M} \& 2 \mathrm{~F})$, the mean difference is defined as "without-exo minus with-exo."

\begin{tabular}{|c|c|c|c|c|c|c|c|c|}
\hline \multirow[b]{2}{*}{$\begin{array}{l}\text { Exoskeleton } \\
\text { Intervention }\end{array}$} & \multicolumn{4}{|c|}{ 0\% BW Lifted } & \multicolumn{4}{|c|}{ 20\% BW Lifted } \\
\hline & Without & With & $\begin{array}{c}\text { Mean } \\
\text { difference } \\
(95 \% \mathrm{CI})\end{array}$ & $\begin{array}{c}\text { P- } \\
\text { value* }^{*}\end{array}$ & Without & With & $\begin{array}{c}\text { Mean } \\
\text { difference } \\
(95 \% \mathrm{CI})\end{array}$ & $\begin{array}{c}\text { P- } \\
\text { value* }\end{array}$ \\
\hline $\begin{array}{l}\text { Normalized } \\
\text { metabolic cost } \\
\text { (Watts/kg) }\end{array}$ & $\begin{array}{c}4.64 \\
(1.03)\end{array}$ & $\begin{array}{c}4.28 \\
(1.06)\end{array}$ & $\begin{array}{c}0.36 \\
(0.2-0.52)\end{array}$ & 0.0003 & $\begin{array}{c}6.50 \\
(1.14)\end{array}$ & $\begin{array}{c}6.07 \\
(1.19)\end{array}$ & $\begin{array}{c}0.43 \\
(0.18-0.69)\end{array}$ & 0.0025 \\
\hline $\begin{array}{c}\text { Average VO2 } \\
\text { consumption } \\
(\mathrm{L} / \mathrm{min})\end{array}$ & $\begin{array}{l}1.08 \\
(0.22)\end{array}$ & $\begin{array}{c}0.98 \\
(0.20)\end{array}$ & $\begin{array}{c}0.097 \\
(0.047- \\
0.148)\end{array}$ & 0.001 & $\begin{array}{c}1.51 \\
(0.25)\end{array}$ & $\begin{array}{c}1.39 \\
(0.25)\end{array}$ & $\begin{array}{c}0.117 \\
(0.049-0.186)\end{array}$ & 0.002 \\
\hline
\end{tabular}




\section{Modeling Results}

We first present the results of modeling the metabolic reductions without an exoskeleton in order to validate our overall modeling approach. Table 2 shows the resulting $K$-values from the best-fit of the experimental results in $(41,81)$ to the model, for stoop and squat lifting at $10 \mathrm{lifts} / \mathrm{min}$. Figure 3 compares the predicted metabolic cost using Equation (1) and the coefficients in Table 2 with the experimental results in (81) and (41). Moreover, Table 3 illustrates the predicted and experimental results in $(41,81)$ and the accuracy of prediction for different external loads at $10 \mathrm{lifts} / \mathrm{min}$. According to Figure 3 , the predicted values are very close to the measured values for all of the external loads. For example, for 1 and $8.5 \mathrm{~kg}$, the prediction error is within $1.8 \%$ of squat lifting and within $0.9 \%$ of stoop lifting. The prediction error is slightly higher for heavier external loads, up to $2.3 \%$ for squat lifting. Thus, by fitting only a few values, our model matches the experimental data for stoop and squat very accurately.

Table 2: Calculated $K$-values for stoop and squat metabolic cost prediction. Note that the $K$-values for stoop and squat were obtained by fitting Equation (1) with the experimental data in Welbergen 1991 (81) ( $\mathrm{N}=9$ Males) and Hagen 1994 (41) ( $\mathrm{N}=10$ Males), while the $K$-values for freestyle lifting were obtained by fitting Equation (2) with the without-exoskeleton data from this experiment $(\mathrm{N}=15 ; 13 \mathrm{M} \& 2 \mathrm{~F})$.

\begin{tabular}{|c|c|c|c|c|c|}
\hline \multicolumn{3}{|c|}{$\boldsymbol{K}_{\text {Body }}$} & \multicolumn{3}{c|}{$\boldsymbol{K}_{\text {Box }}$} \\
\hline Stoop Lifting & Squat Lifting & $\begin{array}{c}\text { Freestyle } \\
\text { Lifting }\end{array}$ & Stoop Lifting & Squat Lifting & $\begin{array}{c}\text { Freestyle } \\
\text { Lifting }\end{array}$ \\
\hline 3.85 & 4.48 & 4.31 & 10.67 & 13.26 & 14.30 \\
\hline
\end{tabular}

Table 3: Predicted metabolic cost vs. experimental results in Welbergen 1991 (81) ( $\mathrm{N}=9$ Males) and Hagen 1994 (41) $(\mathrm{N}=10$ Males) for stoop and squat lifting at 10 lifts/min, and predicted metabolic cost vs. experimental results from the no-exoskeleton condition in this experiment $(\mathrm{N}=15 ; 13 \mathrm{M} \& 2 \mathrm{~F})$. *Note that in this experiment, the $20 \%$ $\mathrm{BW}$ mass of $15.85 \mathrm{~kg}$ was only lifted half of the time, and the other half of the time no box was lifted, for an average mass lifted of $7.93 \mathrm{~kg}$. Also note that in our experiment, the model perfectly fit the data because there were two free parameters in the model $\left(\boldsymbol{K}_{\boldsymbol{B} \boldsymbol{o d y}}, \boldsymbol{K}_{\boldsymbol{B} \boldsymbol{B} \boldsymbol{x}}\right)$ and two measurements $(0 \% \mathrm{BW}$ and $20 \%$ BW, both without the exoskeleton).

\begin{tabular}{|c|c|c|c|c|c|}
\hline Lift Style & Study & $\begin{array}{c}\text { Mass Lifted } \\
{[\mathrm{kg}]}\end{array}$ & $\begin{array}{c}\text { Predicted } \\
\text { Metabolic Cost } \\
\text { [Watts] }\end{array}$ & $\begin{array}{c}\text { Experimental } \\
\text { Metabolic Cost } \\
\text { [Watts] }\end{array}$ & \% Error \\
\hline \multirow{4}{*}{$\begin{array}{c}\text { Stoop } \\
\text { (10 lifts/min) }\end{array}$} & $\begin{array}{c}\text { Welbergen } \\
(1991)\end{array}$ & 19 & 498.67 & 495.11 & 0.72 \\
\hline & \multirow{3}{*}{$\begin{array}{l}\text { Hagen } \\
(1994)\end{array}$} & 1 & 289.18 & 286.66 & 0.88 \\
\hline & & 8.5 & 369.82 & 371.29 & -0.40 \\
\hline & & 17 & 460.74 & 466.84 & -1.31 \\
\hline \multirow{4}{*}{$\begin{array}{c}\text { Squat } \\
\text { (10 lifts/min) }\end{array}$} & $\begin{array}{c}\text { Welbergen } \\
(1991)\end{array}$ & 19 & 647.67 & 662.47 & -2.24 \\
\hline & \multirow{3}{*}{$\begin{array}{l}\text { Hagen } \\
\text { (1994) }\end{array}$} & 1 & 380.75 & 387.67 & -1.79 \\
\hline & & 8.5 & 495.52 & 491.42 & 0.83 \\
\hline & & 17 & 623.06 & 608.81 & 2.34 \\
\hline \multirow{2}{*}{$\begin{array}{c}\text { Freestyle } \\
\text { (12 complete } \\
\text { lifts } / \mathrm{min})\end{array}$} & \multirow{2}{*}{$\begin{array}{l}\text { This } \\
\text { Experiment }\end{array}$} & 0 & 363.8 & 363.80 & 0.00 \\
\hline & & $7.93^{*}$ & 510.9 & 510.88 & 0.00 \\
\hline
\end{tabular}




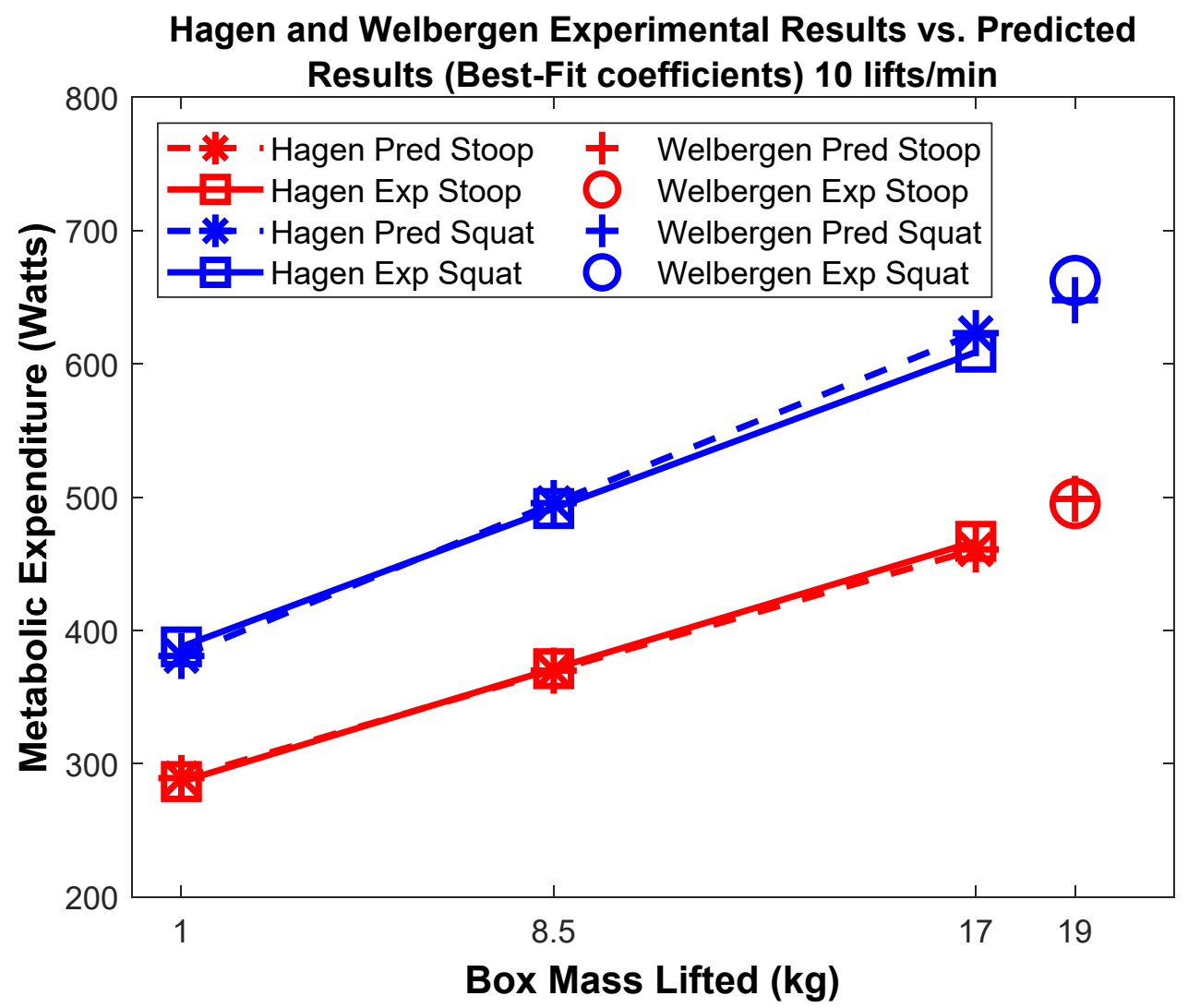

Figure 3: Predicted metabolic cost vs. experimental results in Welbergen 1991 (81) (N = 9 Males) and Hagen 1994 (41) $(\mathrm{N}=10$ Males) for stoop and squat lifting at 10 lifts/min.

Table 2 also shows the resulting $K$-values from the best-fit of our experimental results for freestyle lifting, and Table 3 also shows the predicted and experimental results for the current study. Note that since our without-exoskeleton data consisted of two points (at $0 \% \mathrm{BW}$ and $20 \%$ BW) and there are two parameters being fitted ( $K_{B o d y}$ and $K_{B o x}$ for freestyle lifting), the prediction perfectly matches the experimental data. The resulting $K_{\text {Body }}$ and $K_{B o x}$ for freestyle lifting in our current study are 4.31 and 14.30 , respectively. The $K_{\text {Body }}$ value for freestyle lifting in our study is in between the $K_{B o d y}$ values for stoop and squat (fit to data from other studies), while the $K_{B o x}$ value for freestyle lifting in our study is slightly $(7.8 \%)$ larger than the squat $K_{B o x}$ fit to data from other studies.

Table 4 shows the $K_{E x o, l o w e r}$ and $K_{E x o, l i f t}$ used in predictions for freestyle, stoop, and squat lifting with our exoskeleton under Assumptions 1-4. The measured metabolic cost (freestyle) along with the metabolic cost predictions (stoop, squat and freestyle) are compared in Figure 4 (Assumptions 1 and 2) and Figure 5 (Assumptions 3 and 4), using the $K$-values from Table 4 . In both Figures 4 and 5, the predicted metabolic costs (Assumptions 1 and 2) are shown with dashed lines whereas the experimental metabolic costs are depicted with solid lines. As shown in Figure 4, Assumptions 1 and 2 led to a very similar predicted metabolic cost values for each lifting type (see the percent difference in Table 5). This 
was also the same in Figure 5 where the predicted metabolic cost using Assumptions 3 and 4 for each lifting type were close to each other. This was expected as the mechanical efficiency of our exoskeleton in energy storage and return is close to one $\left(\eta_{\text {exo }}=0.95\right)(19)$. For freestyle lifting in with-exoskeleton conditions, the predicted (i.e. with four assumptions explained above) and experimental metabolic cost values plus the corresponding percentage errors are summarized in Table 5. According to data collected by Chang and colleagues, the corresponding mean [range of $25^{\text {th }}$ to $75^{\text {th }}$ percentile] of $X$ (i.e., the mechanical work that exoskeleton stores during bending) for freestyle, squat, and stoop lifting were found to be 59.3 [42.2-73.1], 51.5 [33.4-65.6], and 61.8 [44.9-79.7] Joules, respectively (see Table 3 in (19) for mean values of $X$ ). Since we did not directly measure $X$ in our current experiment, we assumed that $X$ could vary between the $25^{\text {th }}$ and $75^{\text {th }}$ percentile for each lifting type and hence we provided the corresponding metabolic cost predictions in Table 5. These ranges provide a sensitivity analysis for the prediction results.

Table 4: Assumptions about values of $K_{E x o, l o w e r}$ and $K_{E x o, l i f t}$ used in subsequent calculations for this experiment $(\mathrm{N}=15 ; 13 \mathrm{M} \& 2 \mathrm{~F})$.

\begin{tabular}{|c|c|c|c|c|c|}
\hline $\begin{array}{c}\text { Lifting } \\
\text { Type }\end{array}$ & Assumption & Sum & Ratio & $K_{\text {Exo,lower }}$ & $K_{E x o, l i f t}$ \\
\hline \multirow{4}{*}{ Freestyle } & 1 & $K_{\text {Exo,lower }}+K_{\text {Exo,lift }}=K_{\text {Body }}$ & $K_{\text {Exo,lower }}=K_{\text {Exo,lift }}$ & 2.16 & 2.16 \\
\hline & 2 & $K_{\text {Exo,lower }}+K_{\text {Exo,lift }}=K_{\text {Body }}$ & $K_{\text {Exo,lower }}=0.25 K_{\text {Exo,lift }}$ & 0.86 & 3.45 \\
\hline & 3 & $K_{E x o, \text { lower }}+K_{E x o, \text { lift }}=K_{\text {Box }}$ & $K_{\text {Exo,lower }}=K_{\text {Exo,lift }}$ & 7.15 & 7.15 \\
\hline & 4 & $K_{E x o, \text { lower }}+K_{E x o, \text { lift }}=K_{B o x}$ & $K_{\text {Exo,lower }}=0.25 K_{\text {Exo,lift }}$ & 2.86 & 11.44 \\
\hline \multirow{4}{*}{ Squat } & 1 & $K_{\text {Exo,lower }}+K_{E x o, \text { lift }}=K_{\text {Body }}$ & $K_{\text {Exo,lower }}=K_{\text {Exo,lift }}$ & 2.24 & 2.24 \\
\hline & 2 & $K_{\text {Exo,lower }}+K_{\text {Exo,lift }}=K_{\text {Body }}$ & $K_{\text {Exo,lower }}=0.25 K_{\text {Exo,lift }}$ & 0.90 & 3.58 \\
\hline & 3 & $K_{E x o, \text { lower }}+K_{E x o, \text { lift }}=K_{B o x}$ & $K_{\text {Exo,lower }}=K_{\text {Exo,lift }}$ & 6.63 & 6.63 \\
\hline & 4 & $K_{E x o, \text { lower }}+K_{E x o, \text { lift }}=K_{B o x}$ & $K_{\text {Exo,lower }}=0.25 K_{\text {Exo,lift }}$ & 2.65 & 10.61 \\
\hline \multirow{4}{*}{ Stoop } & 1 & $K_{\text {Exo,lower }}+K_{\text {Exo,lift }}=K_{\text {Body }}$ & $K_{\text {Exo,lower }}=K_{\text {Exo,lift }}$ & 1.93 & 1.93 \\
\hline & 2 & $K_{\text {Exo,lower }}+K_{\text {Exo,lift }}=K_{\text {Body }}$ & $K_{\text {Exo,lower }}=0.25 K_{\text {Exo,lift }}$ & 0.77 & 3.08 \\
\hline & 3 & $K_{E x o, \text { lower }}+K_{E x o, \text { lift }}=K_{B o x}$ & $K_{\text {Exo,lower }}=K_{\text {Exo,lift }}$ & 5.33 & 5.33 \\
\hline & 4 & $K_{E x o, \text { lower }}+K_{E x o, \text { lift }}=K_{B o x}$ & $K_{\text {Exo,lower }}=0.25 K_{\text {Exo,lift }}$ & 2.13 & 8.53 \\
\hline
\end{tabular}




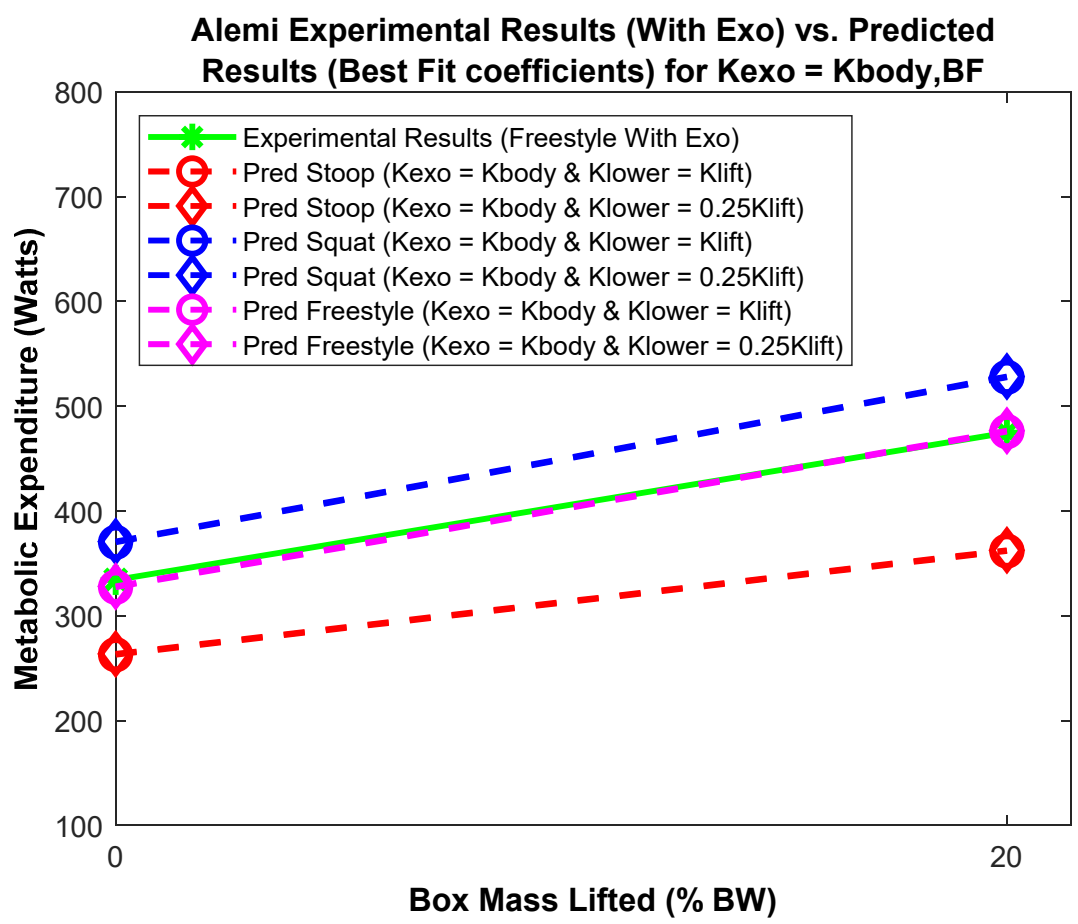

Figure 4: Measured metabolic cost (freestyle; $\mathrm{N}=15 ; 13 \mathrm{M} \& 2 \mathrm{~F}$ ) and predicted metabolic cost (stoop, squat and freestyle) for two box weights ( $0 \%$ and $20 \%$ BW) using Assumptions 1 and 2 (Assumption 1: exoskeleton's $K$ values are equal to body $K$-values while $K_{\text {Body,lower }} \approx K_{\text {Body,lift }}$; Assumption 2: exoskeleton's $K$-values are equal to body $K$-values while $K_{\text {Body,lower }} \approx 0.25 K_{\text {Body,lift }}$ )

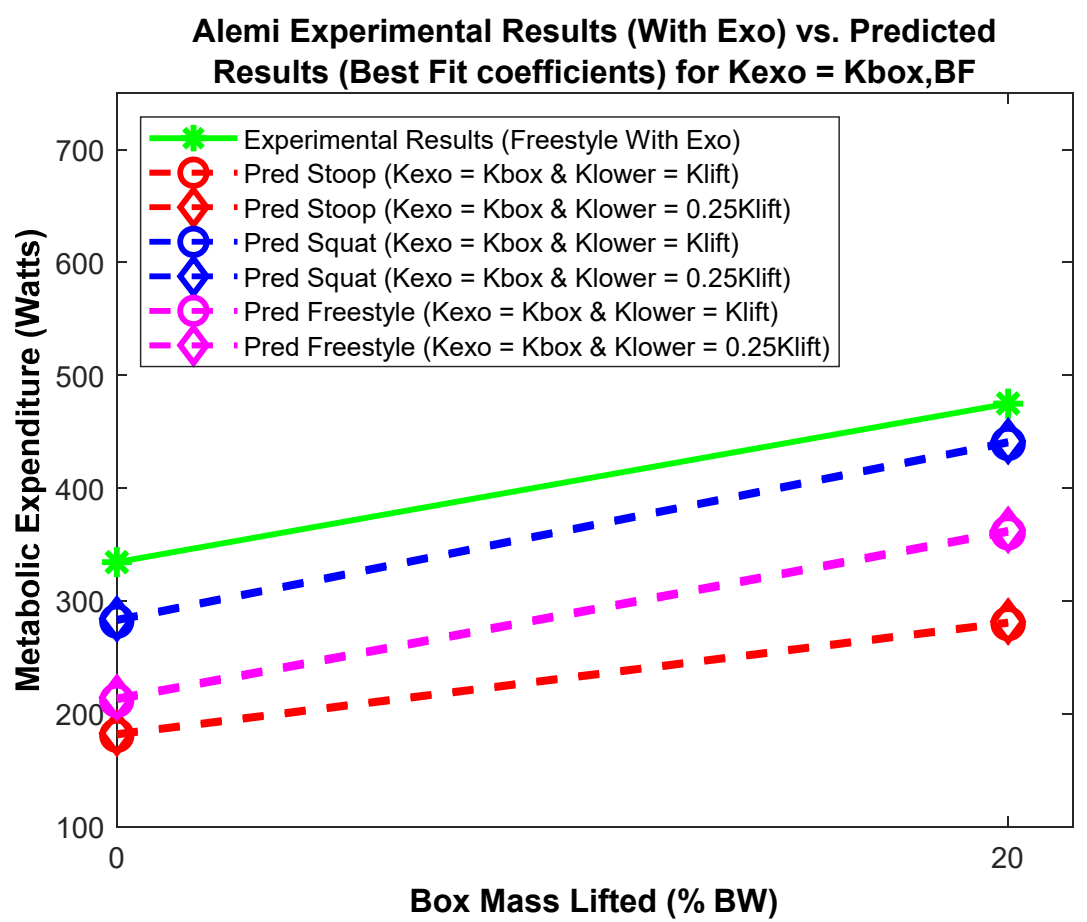

Figure 5: Measured metabolic cost (freestyle; $\mathrm{N}=15 ; 13 \mathrm{M} \& 2 \mathrm{~F}$ ) and predicted metabolic cost (stoop, squat and freestyle) for two box weights ( $0 \%$ and $20 \%$ BW) using Assumptions 3 and 4 (Assumption 3: exoskeleton's $K$ values are equal to box $K$-values while $K_{\text {Box,lower }} \approx K_{B o x, \text { lift }}$; Assumption 4: exoskeleton's $K$-values are equal to box $K$-values while $K_{B o x, \text { lower }} \approx 0.25 K_{\text {Box,lift }}$ ) 
Table 5: Experimental vs. predicted metabolic costs of lifting with the exoskeleton (at 6 lifts $/ \mathrm{min}$ ) for two box weights $(0 \%$ and $20 \%$ of $\mathrm{BW})(\mathrm{N}=15 ; 13 \mathrm{M} \& 2 \mathrm{~F})$. The different prediction estimates are based on the $25^{\text {th }}$ percentile, mean, and $75^{\text {th }}$ percentile of exoskeleton stored energy in (19), as a sensitivity analysis for the model.

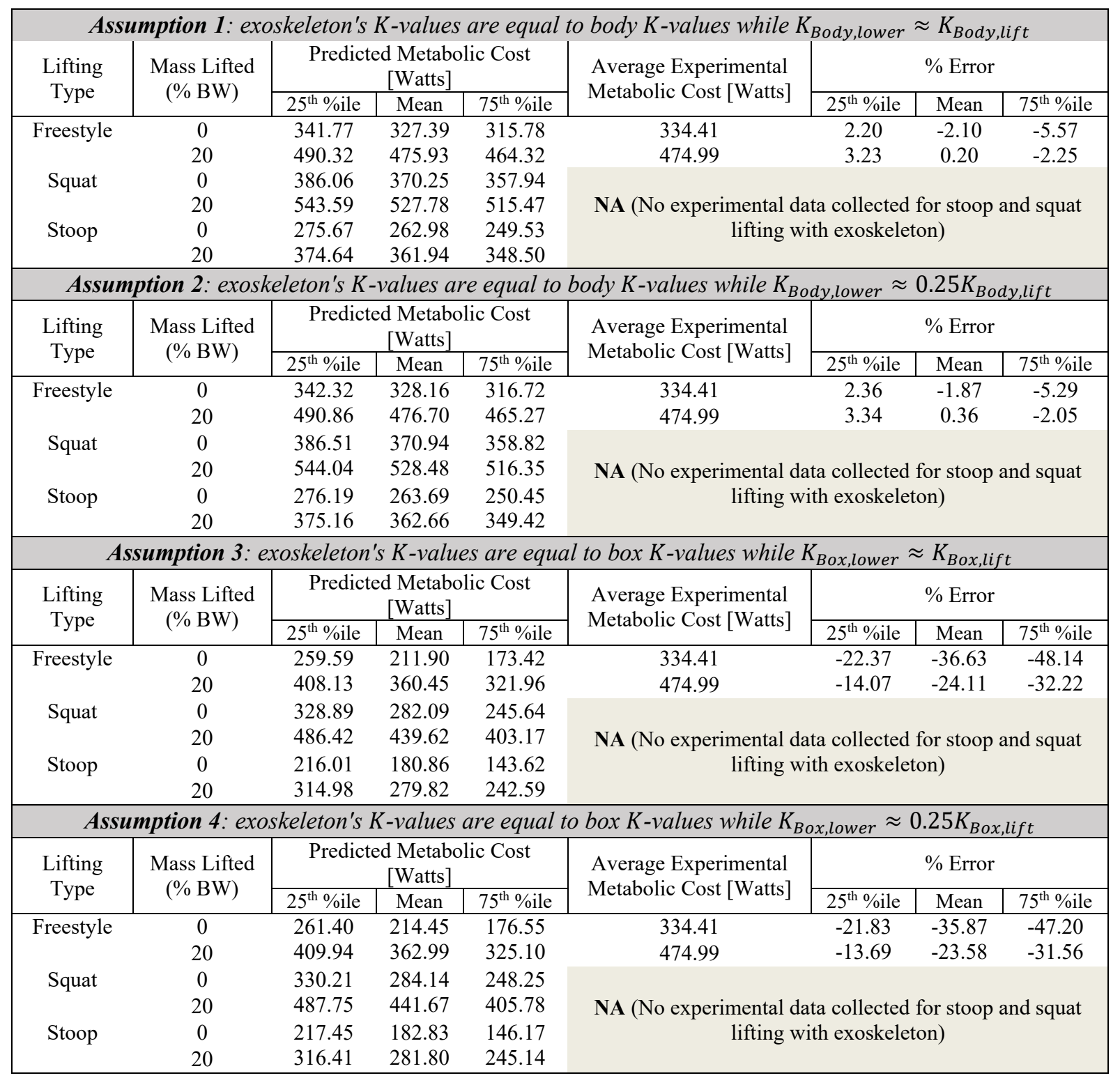

\section{Discussion}

The values of $K_{B o d y}$ and $K_{B o x}$ are effectively the inverse of the biomechanical efficiencies of lifting the body and box, respectively, taking into account the entire body's motion including the contributions of passive structures. Computing $1 / K_{\text {Body }}$ results in $0.26,0.22$, and 0.23 for stoop, squat, and freestyle, respectively, while computing $1 / K_{B o x}$ results in $0.094,0.075$, and 0.070 for stoop, squat, and freestyle, respectively. The values found for $1 / K_{\text {Body }}$ are largely similar to efficiencies found in previous studies: Gaesser and Brooks found work efficiencies between 0.19 and 0.27 for cycling, depending on the torque and speed (29); Ryschon et al. found efficiencies during cycling of 0.347 for eccentric force production and 
0.150 for concentric force production, which average to 0.249 (68); Kamon found that climbing a ladder has an efficiency of 0.257 and descending one has an efficiency of 0.976 (46). A number of studies have examined the overall metabolic efficiency of lifting boxes, but these are not directly comparable as they combine the efficiencies of the body and box $(25,42,69,81)$, and relatively few other studies include lifting very light boxes which would only include the body term.

The efficiency values corresponding to $1 / K_{\text {Body }}$ appear to be $\sim 3$ times that of $1 / K_{B o x}$. The lower efficiency of $K_{B o x}$ could be due to needing to recruit more muscles to lift a load some distance from the body, co-contraction to maintain stability, or a decreased ability to store energy in elastic structures in the body when lifting a mass with the hands. We speculate that there could be a relatively wide range of possible values for both $K_{B o d y}$ and $K_{B o x}$ depending on the specific lifting motions and posture. Prior work has shown that the metabolic cost of lifting boxes depends on the specific box shape (9) and also if the person is lifting a bag or a box (45), which should influence $K_{B o x}$. Similarly, we expect that $K_{B o d y}$ will vary depending on if a person keeps their back straight or if they use passive structures in their back to support the load, on the extent to which they co-contract their muscles, and on the distribution of weight over their body; Garg found that males and females can have different lifting efficiencies (32). Trained lifters also likely have techniques to reduce the energetic cost of lifting. Finally, we note that these values may vary based on the lifting speed, particularly as lifts are done faster and the effects of the body's inertia may become more significant.

Comparing the freestyle $K$-values fit to our current study with the $K$-values obtained from fitting the model to prior studies that did stoop and squat lifting $(41,81)$, the freestyle $K_{B o d y}$ is between stoop and squat $K_{B o d y}$, which is what we would expect since freestyle lifting kinematics are in between those of stoop and squat. However, the freestyle $K_{B o x}$ is $7.8 \%$ larger than the squat $K_{B o x}$; we would also expect this value to be between stoop and squat. Our larger value might be because in both (41) and (81), professional subjects were recruited for the experiments, whereas our experiment used a combination of experienced and novice lifters, which may result in some differences in lifting efficiency.

As it is clearly evident in Figures 4 and 5, the metabolic cost prediction of freestyle lifting using Assumptions 1 and $2\left(K_{E x o} \approx K_{B o d y}\right)$ is a much better estimate compared to the same prediction using Assumptions 3 and $4\left(K_{E x o} \approx K_{B o x}\right)$. According to Table 5, the absolute estimation errors for freestyle prediction with Assumptions 1 and 2 are within $2.1 \%$ for $0 \%$ of BW and $0.4 \%$ for $20 \%$ of BW. However, using Assumptions 3 and 4 led to much higher prediction errors of up to $36.6 \%$ for $0 \%$ of BW and $24.1 \%$ for $20 \%$ of BW. Even if the stored energy $(X)$ in this experiment was in the $25^{\text {th }}$ or $75^{\text {th }}$ percentile of that measured in (19), Assumptions 1 and 2 still lead to vastly better predictions than Assumptions 3 and 4. According to Assumptions 1 and 2, the average predicted change in metabolic cost due to the exoskeleton, as calculated with Equation $5 \mathrm{~b}$, is about 35.3 Watts whereas the corresponding average measured change in metabolic cost is 32.6 Watts. This $8.3 \%$ difference is likely due to the fact that subjects received minimal training and adaptation to the exoskeleton before the test, although it could be due to the exoskeleton storing less energy than expected.

Assuming that the exoskeleton's $K$-values equal the body $K$-values makes intuitive sense, since the exoskeleton is worn over the body and does not substantially change the body's kinematics, but instead augments the joint torques at the hip and lower back. In the limit of an exoskeleton that completely offsets the weight of the torso during stoop lifting, $\left(X=\left|W_{B o d y}\right|\right.$ in Equation $\left.3 \mathrm{~b}\right)$, and with perfect energy return ( $\eta_{\text {exo }}=1$ in Equation $3 b$ ), using $K_{\text {Exo }} \approx K_{\text {Body }}$ will result in canceling the body term exactly, while using 
$K_{E x o} \approx K_{B o x}$ will lead to a theoretically negative metabolic costs, which is of course not correct. Therefore, $K_{E x o} \approx K_{B o x}$ is not a good assumption.

In the model, a consequence of assuming that the body moves quasi-statically is that an exoskeleton that perfectly offsets the weight of the torso, with perfect efficiency, would lead to no net metabolic expenditure during stoop lifting. This is also clearly not the case, since lifting is not quasi-static and the body must accelerate and decelerate its mass and that of the box. Since the original $K_{B o d y}$ and $K_{B o x}$ factors lump together the contributions from quasi-static changes in potential energy as well as the dynamic effects, it is reasonable that the $K_{E x o}$ factors would in fact be less than both of these, i.e. $K_{E x o} \approx C K_{\text {Body }}$ where $C$ is a coefficient that is the contribution due to inertial effects and is less than one. The specific value of $C$ is likely between $0.93-0.97$ for normal lifting speeds based on the fraction of total energy caused by the peak kinetic energy, as previously discussed $(67,72)$. With a value of $C$ in this range, so that $K_{E x o}<K_{B o d y}$, our measured metabolic reductions would correspond even more closely to the predicted value. It is interesting that the observed metabolic cost reduction for $0 \%$ and $20 \%$ of BW are close to each other (absolute difference $\sim 6.5$ Watts) and thus appear to be independent of the external load. This is also predicted by Equation $5 \mathrm{~b}$, where $\Delta M_{\text {Lifting,Exo }}$ does not depends on $\left|W_{B o x}\right|$ and only depends on lifting frequency $(L)$, $K_{\text {Body }}, K_{\text {Exo }},\left|W_{\text {Exomass }}\right|, X$, and $\eta_{\text {exo }}$. This result lends support to our model being accurate.

If the exoskeleton's $K$-values equal the body $K$-values, then the metabolic benefit of a passive back support exoskeleton is not equivalent to having a "negative weight" box. Furthermore, since the $K_{B o x}$ values are so much larger than $K_{B o d y}$ values, it seems that the dynamics of lifting are different when a person is lifting a box versus just their body. We speculate that if a person wore additional mass that was closefitting over their body, the value of $K_{\text {ExtraMass }}$ would be close to $K_{B o d y}$ instead of $K_{B o x}$. Consequently, since the exoskeleton is form-fitting over the body, then it is equivalent to having a negative mass that is form-fitting over the body.

The other important concern is to judge how useful it is to have an exoskeleton with a high energy return, given our inability to determine the ratio of $R_{E x o}=\frac{K_{E x o, l i f t}}{K_{E x o, l o w e r}}$. If the ratio is between 2 to 4 , then the value of $\eta_{\text {exo }}$ is very crucial because the metabolic cost of the lifting phase costs two to four times more than the metabolic cost in the lowering phase. To gain additional insight into this value, we fit our model to data in (22) and (30). In both of these experiments, participants either lifted or lowered a box, and the box was returned to its original height by other means (a powered mechanism or another person, respectively); details of the model fits and results are summarized in Appendix $\mathrm{C}$. These experiments gave values of the ratio $R_{B o x}=\frac{K_{B o x, \text { lift }}}{K_{B o x, \text { lower }}}$ between 1.68 and 5.29. While $R_{E x o}$ is distinct from $R_{B o x}$, and $R_{E x o}$ is likely much closer to $R_{B o d y}=\frac{K_{\text {Body,lift }}}{K_{\text {Body,lower }}}$, the ratio between the lifting and lowering efficiency is probably similar for the body, a lifted mass, and the exoskeleton since these are all related to the efficiency of concentric versus eccentric muscle contraction. While the experiments we fit to find $R_{B o x}$ used a small number of participants (6 and 3, respectively, in (22) and (30)) and resulted in a wide range of values, it appears that $R_{B o x}$ is definitely greater than one. Further experimentation with a larger number of subjects will give additional insight into $R_{B o x}$, and experiments with an active exoskeleton that helps with either lifting or lowering will give insight into $R_{E x o}$. 
Even with a rough estimate of the ratio, we can gain insight into how much an exoskeleton's efficiency matters. Supposing that $K_{\text {Exo,lift }} \approx 3 K_{\text {Exo,lower }}$, if our exoskeleton efficiency was 0.8 or 0.5 instead of 0.95 , we would expect metabolic reductions of 28.6 or 17.0 Watts instead of 34.3 Watts, respectively (these values are $83.2 \%$ and $49.7 \%$ of 34.3 Watts, respectively). Hence, if $R_{E x o} \sim 3$, any reductions in exoskeleton efficiency almost directly translate into reductions in metabolic benefit, so it is very important to have an exoskeleton with high energy return.

It should be noted that our study and modeling had several limitations. In the study, there was a relatively large variation in the metabolic results, even without the exoskeleton. This may have been due to the fact that most participants were not experienced lifters. Also, participants had limited time to adapt to the exoskeleton, which may have affected the resulting metabolic reduction. Indeed, two participants had metabolic reductions close to zero, while the maximum reductions in metabolic cost and oxygen consumption achieved by a participant were approximately $200 \%$ higher than the mean values. Additional adaptation time may reduce the variation in measurements and result in a more accurate estimate of metabolic reduction. Additionally, the exoskeleton was not adjusted in strength to the different participants, and may have been too strong for the lightest participants given the short adaptation time. The modeling is also limited in that the $K$-values were calculated only for around 10 lifts/minute, and for lifts that were all roughly from the same starting height to knuckle height. The specific $K$-values and dynamics of lifting may be different for different lifting speeds or for lifting from or to different heights. Relatedly, we did not explicitly include terms in our model for the kinetic energy generated during a lift. However, even despite these limitations, the model is useful for making predictions about the effects of an exoskeleton on the metabolic cost of lifting.

As discussed in the introduction, several earlier studies have already shown the capability of passive back support exoskeletons in reducing the activity of back and leg muscles during lifting tasks. However, very few studies are available that have quantified the metabolic savings that passive BSEs would provide for the wearers during lifting tasks $(5,7,10,11,56,82)$. Those studies reported that using passive BSEs could reduce the metabolic demands by up to $17 \%$ during symmetric $(7,10,56)$ and by $5-6 \%$ during asymmetric (7) lifting tasks. Historically, Whitfield et al. published the very first study exploring the effect of an exoskeleton (i.e., PLAD) on oxygen consumption during symmetric lifting tasks. They concluded that use of PLAD had no significant effects on oxygen consumption, although significant reductions in activity of back muscles were reported (82). Recently, Alemi et al. showed that use of two passive BSEs (i.e., Laevo $^{\mathrm{TM}}$ and Suit ${ }^{\mathrm{TM}}$ ) reduced the metabolic costs by $8.9 \%$ and $12.6 \%$, respectively during repetitive symmetric lifting tasks ( $6.8 \mathrm{~kg}$ load, $5 \mathrm{mins}$ of lifting at $5 \mathrm{lift} / \mathrm{min}$, see Appendix $\mathrm{C}$ in (7)). Additionally, Madinei et al. also conducted a different lifting experiment with Laevo ${ }^{\mathrm{TM}}$ and Suit ${ }^{\mathrm{TM}}$ and they showed that $9.5 \%$ and $13.6 \%$ metabolic savings provided by Laevo ${ }^{\mathrm{TM}}$ and Suit $\mathrm{X}^{\mathrm{TM}}$, respectively, during symmetric lifting tasks in a standing posture (10\% BW, 4 mins of lifting at $10 \mathrm{lift} / \mathrm{min})(56)$. Lately, Baltrusch and colleagues performed two experiments to measure the "net" metabolic reductions (i.e., subtracting the resting metabolic cost from the total metabolic cost) provided by Laevo ${ }^{\mathrm{TM}}$ and SPEXOR during lifting tasks $(10,11)$. In their first study, they showed that the net metabolic demands were reduced by $8 \%$ with the lowcam Laevo $^{\mathrm{TM}}$ (i.e., Laevo ${ }^{\mathrm{TM}}$ supports the wearer at bending angles larger than $20^{\circ}$ ) and $16 \%$ with the highcam Laevo $^{\mathrm{TM}}$ (i.e., Laevo ${ }^{\mathrm{TM}}$ supports the wearer at bending angles from $0-20^{\circ}$ ) during symmetric lifting from ankle height (10 kg load, 5 mins of lifting at 6 lift/min). In their second study, Baltrusch et al. quantified the muscle activity and net metabolic cost reductions provided by the SPEXOR exoskeleton and 
they reported that SPEXOR provided a $\sim 16 \%$ reduction in activity of back muscles and $\sim 18 \%$ reduction in net metabolic demands during repetitive lifting tasks (10 kg load, 5 mins of lifting at 8 lift $/ \mathrm{min}$ ) (11).

The results of our current study showed that VT-Lowe's exoskeleton significantly reduced the total metabolic costs by $\sim 8 \%$ and $\sim 7 \%$ when lifting $0 \%$ (i.e., empty box) and $20 \%$ of BW (5). The resultant net metabolic cost reductions are $\sim 12 \%$ and $\sim 9 \%$ for an empty box and $20 \%$ BW box, respectively. Therefore, our measured metabolic cost reductions are similar in magnitude to the results reported in (7) and (56) where they measured the metabolic savings of the Laevo ${ }^{\mathrm{TM}}$ and SuitX BackX ${ }^{\mathrm{TM}}$ during repetitive lifting tasks. The net metabolic cost reduction of the VT-Lowe's exoskeleton $(\sim 10 \%)$ is also comparable to the corresponding metabolic savings reported in (10) for the low-cam Laevo ${ }^{\mathrm{TM}}(\sim 8 \%)$. In comparison with SPEXOR, the VT-Lowe's exoskeleton showed a lower reduction in metabolic cost. This discrepancy could be due to several factors such as lifting frequency ( $6 \mathrm{lifts} / \mathrm{min}$ vs. $8 \mathrm{lifts} / \mathrm{min})$, lifted weight ( $0 \%$ and $20 \%$ of BW vs. $10 \mathrm{~kg}$ ), task duration (12 min vs. $5 \mathrm{~min}$ ), not having optimized the amount of support for the VTLowe's exoskeleton, different freestyle lifting techniques performed by each participant, insufficient amounts of training, and exoskeleton design features.

In general, we believe that 30-minute training with an exoskeleton is not enough time for suitable adaptation to an exoskeleton, although at this stage, it is still unknown how long of a training would be sufficient for full adaptation. Further work is necessary to develop a standardized methodology for exploring the dependence of metabolic cost on specific BSE design approaches (e.g., the torque-angle relationship) during various lifting tasks.

\section{Conclusions}

In this study, the metabolic cost and oxygen consumption results supported the hypothesis that the VT-Lowe's exoskeleton could reduce the metabolic demands and oxygen uptake during freestyle lifting. The exoskeleton revealed a significant metabolic reduction of $8 \%$ when lifting an empty box and $7 \%$ when lifting $20 \%$ of a person's body weight. In general, reductions in both metabolic expenditure and oxygen consumption lower the possibility of the fatigue for manual material handling workers and consequently, the exoskeleton will reduce the risk of injuries especially for tasks involving heavy or repeated lifting. Moreover, the reduction in the activity of back and leg muscles as reported in (6) combined with the reduction in metabolic cost and oxygen uptake from this paper present the VT-Lowe's exoskeleton as a promising passive back support exoskeleton.

We also presented models of the metabolic cost reduction due to a passive BSE. Initially a metabolic cost prediction model was developed for without-exoskeleton conditions and the model was fitted with the experimental data in (81) and (41) and validated for stoop and squat lifting, at $10 \mathrm{lifts} / \mathrm{min}$. Next, the prediction model was modified and fitted to the experimental results of the without-exoskeleton conditions in this study to calculate the effective biomechanical efficiency of box and body for freestyle lifting. The metabolic cost model was again modified and some terms were added to reflect the effect of an exoskeleton on metabolic cost. For metabolic cost prediction of with-exoskeleton conditions, four assumptions were made to calculate the exoskeleton's $K$-values. The modeling results demonstrated that much smaller estimation errors were obtained when the exoskeleton's $K$-values were set to be approximately equal to $K_{B o d y}$.

Of course, the specific $K$-values in the metabolic prediction model may need to be modified for each experimental design based on the specific lifting mechanics and other possible task elements in the experiment. Nevertheless, the prediction model provides a roadmap to improve our understanding about 
the factors that significantly contribute to the metabolic cost savings from an exoskeleton, and its use can lead to improved designs for future lifting exoskeletons. While energy savings from metabolic reductions are important, a passive lifting exoskeleton's potential to reduce injuries (due to both reductions in muscle activity and metabolic fatigue) will likely be the most important benefit to the individuals wearing it.

\section{Disclosures}

This work was funded by Lowe's, Inc. The study sponsors had no involvement in the experimental design or the collection, analysis, and interpretation of the data, in the writing of the manuscript, or in the decision to submit the manuscript for publication. A. Asbeck and J. Geissinger are co-authors on a patent for the exoskeleton.

\section{Authorship Contributions}

Conceptualization: M. M. Alemi, A. T. Asbeck; Experimental Design/Methodology: M. M. Alemi, A. T. Asbeck; Human Subject Testing: M. M. Alemi, A. A. Simon, J. Geissinger; Formal Analysis: M. M. Alemi, A. T. Asbeck; Metabolic Cost Coding/Modeling: M. M. Alemi, A. T. Asbeck; Supervision: A. T. Asbeck; Writing - original draft: M. M. Alemi, A. T. Asbeck; Writing - review \& editing: M. M. Alemi, A. T. Asbeck

\section{Endnote}

The raw metabolic data and scripts used to fit the model to the data and perform predictions can be found here: DOI: $10.6084 / \mathrm{m} 9$.figshare.c.5488929

\section{Appendix A}

Table A: Average oxygen consumption and metabolic cost in Hagen 1994 (41) ( $\mathrm{N}=10$ Males) and Welbergen 1991 (81) $(\mathrm{N}=9$ Males) for stoop and squat lifting at $10 \mathrm{lifts} / \mathrm{min}$. Note that to calculate the metabolic cost from oxygen consumptions, it was assumed that $1 \mathrm{~L} \mathrm{O} 2 / \mathrm{min}=348.67$ Watts.

\begin{tabular}{|c|c|c|c|c|}
\hline Study & Lifting Type & Mass Lifted [kg] & Oxygen Consumption & Metabolic Cost [W] \\
\hline \multirow{3}{*}{ Hagen (1994) } & \multirow{3}{*}{ Stoop } & 1 & $10.5[\mathrm{ml} /(\mathrm{kg} \cdot \mathrm{min})]$ & 286.66 \\
\cline { 3 - 5 } & & 8.5 & $13.6[\mathrm{ml} /(\mathrm{kg} \cdot \mathrm{min})]$ & 371.29 \\
\cline { 3 - 5 } & \multirow{3}{*}{ Squat } & 17 & $17.1[\mathrm{ml} /(\mathrm{kg} \cdot \mathrm{min})]$ & 466.84 \\
\cline { 3 - 5 } & & 1 & $14.2[\mathrm{ml} /(\mathrm{kg} \cdot \mathrm{min})]$ & 387.67 \\
\cline { 3 - 5 } & & 8.5 & $18[\mathrm{ml} /(\mathrm{kg} \cdot \mathrm{min})]$ & 491.42 \\
\cline { 3 - 5 } & & 17 & $22.3[\mathrm{ml} /(\mathrm{kg} \cdot \mathrm{min})]$ & 608.81 \\
\hline \multirow{2}{*}{ Welbergen (1991) } & Stoop & 19 & $1.42[\mathrm{~L} / \mathrm{min}]$ & 495.11 \\
\cline { 3 - 5 } & Squat & 19 & $1.9[\mathrm{~L} / \mathrm{min}]$ & 662.47 \\
\hline
\end{tabular}


Table B: Various parameters used to validate and predict the metabolic cost (i.e., energy expenditure) in Hagen 1994 (41) ( $\mathrm{N}=10$ Males), Welbergen 1991 (81) ( $\mathrm{N}=9$ Males), and current study (without and with exoskeleton, $\mathrm{N}$ $=15 ; 13 \mathrm{M} \& 2 \mathrm{~F})$. Ranges of values are given for the change in Body COM; these correspond to the height changes for an empty box and a $20 \%$ BW box, respectively. *In this experiment, the box was not held during half of the cycles, whereas in the other experiments it was held continuously. 12 complete cycles per minute is equivalent to the other studies when an empty box was used in this experiment. In the equations in this paper, $\mathrm{L}=6$ was used since the equations include both the with-box and without-box portions. **The changes in body COM height for stoop and squat were only calculated for comparison to freestyle lifts.

\begin{tabular}{|c|c|c|c|}
\hline Parameters & $\begin{array}{c}\text { Hagen } \\
\mathbf{( 1 9 9 4 )}\end{array}$ & $\begin{array}{c}\text { Welbergen } \\
\mathbf{( 1 9 9 1 )}\end{array}$ & $\begin{array}{c}\text { Current Study } \\
\text { (Without \&With Exo) }\end{array}$ \\
\hline Avg. Body Mass [kg] & 78.30 & 77.20 & 79.27 \\
\hline Avg. Body Height [m] & 1.80 & 1.82 & 1.74 \\
\hline Mass Lifted [kg] & $1,8.5,17$ & 19 & $\begin{array}{c}0,20 \% \text { BW }= \\
0,15.85^{*} \mathrm{~kg}\end{array}$ \\
\hline Lifting Frequency [lifts/min] & $\begin{array}{c}10 \text { complete } \\
\text { cycles/min }\end{array}$ & $\begin{array}{c}10 \text { complete } \\
\text { cycles/min }\end{array}$ & $\begin{array}{c}12 \text { complete } \\
\text { cycles/min* }\end{array}$ \\
\hline Knuckle Height [m] & 0.799 & unknown & 0.770 \\
\hline Box Handle Height [m] & 0.21 & unknown & 0.225 \\
\hline Standing Metabolic Cost [W] & 125.00 & 123.24 & 126.55 \\
\hline Change in Box COM [m] & 0.589 & 0.663 (stoop), & $0.642(\mathrm{squat})$ \\
\hline Change in Body COM [stoop] [m] & $0.312-0.327$ & 0.320 & 0.545 \\
\hline Change in Body COM [squat] [m] & $0.424-0.490$ & 0.460 & $0.289-0.302^{* *}$ \\
\hline Change in Body COM [freestyle] [m] & - & - & $0.354-0.393^{* *}$ \\
\hline
\end{tabular}

Table C: Best fit equation relating stature and knuckle height for males, fit to the ANSUR1 data from (38) ( $\mathrm{n}=1774$ male subjects). Both Stature and Knuckle Height are in meters.

\section{Best Fit Line Equation [m]}

Knuckle Height $=(0.5074 \times$ Stature $)-0.11414$

Table D: Equations relating the normalized change in height of a box to the normalized change in height of the body's COM. In the equations below, X refers to the normalized box height change (box height/person height) while $\mathrm{Y}$ refers to the normalized full-body COM height change (i.e., COM height/person height). These equations were used to calculate the vertical displacement of body COM for different lifting types (stoop, squat and freestyle) and box weights $(0 \%$ and $20 \% \mathrm{BW})$. In this paper, the $0 \% \mathrm{BW}$ equations were used for empty boxes or boxes less than or equal to $2.7 \mathrm{~kg}$, the $20 \% \mathrm{BW}$ equations were used for box masses equal to or greater than $20 \% \mathrm{BW}$, and linear interpolation between the $0 \% \mathrm{BW}$ equations and $20 \% \mathrm{BW}$ equations was done for masses between $2.7 \mathrm{~kg}$ and $20 \% \mathrm{BW}$. The equations are taken from kinematics data measured in (72).

\begin{tabular}{|c|c|c|c|}
\hline Best Fit Line Equation & Stoop & Squat & Freestyle \\
\hline Lifting 0\% BW & $\mathrm{Y}=0.5297 \mathrm{X}$ & $\mathrm{Y}=0.7192 \mathrm{X}$ & $\mathrm{Y}=0.6485 \mathrm{X}$ \\
\hline Lifting $20 \% \mathrm{BW}$ & $\mathrm{Y}=0.5543 \mathrm{X}$ & $\mathrm{Y}=0.8315 \mathrm{X}$ & $\mathrm{Y}=0.7191 \mathrm{X}$ \\
\hline
\end{tabular}

The knuckle height in Table B is calculated using a best-fit line to the raw data for males reported in (38) (Table C). The equation in Table $\mathrm{C}$ was used to calculate the knuckle height based on the corresponding average stature of the study if the actual knuckle height was not available. 
Box handle height in Table B is the vertical distance between the bottom of the lifted weight and the weight handle. For this study and (41), the change in box COM is calculated by subtracting the handle height from the calculated knuckle height. Note that the knuckle height and box handle height were not known or needed in (81) since the paper reported the change in body heights directly, and the change in box heights was calculated based on other data in the paper.

The change in vertical displacement of body COM for each lifting type is calculated using the equations in Table D. The equations in Table D provide the best fit lines which relate the normalized body's COM height to the normalized change in box height lifting (note that body's COM height and change in box height were normalized with respect to person's height). The equations in the table were calculated from kinematics measurements in (72).

\section{Appendix B}

In this Appendix we discuss the physics of lowering and raising up while wearing an exoskeleton during a lift, and specifically how an exoskeleton can cause a metabolic reduction while lowering. To analyze this, we first present raw data of the EMG of the back and stomach muscles during a complete lifting cycle similar to that performed in this paper. This data is from the experiment conducted in (6), which used the same exoskeleton and lifting pattern as in this paper, but with four complete lifting cycles per minute instead of six as in this paper. The EMG data is normalized by the maximum voluntary contraction (MVC), which was obtained by the subjects performing a series of isometric contractions against bracing that isolated the specific muscles. Verbal encouragement was used during the contractions to obtain higher muscle activity. After filtering the signals as detailed in (6), the peaks were used as the MVC values. Both the without-exo and with-exo trials were normalized by the same MVC values.

Figure A shows representative lifting cycles from two participants doing Stoop lifts, while Figure B shows representative lifting cycles from the same two participants doing Freestyle lifts. In each case, the participants lifted $20 \%$ of their bodyweight. In the graphs, P1 was a male, $1.76 \mathrm{~m}$ tall and with a body mass of $82.3 \mathrm{~kg}$, while P2 was a male, $1.69 \mathrm{~m}$ tall and with a mass of $73.3 \mathrm{~kg}$. EMG signals were used from these participants were selected because they had particularly clean (noise-free) EMG signals which are easier to interpret. In the experiment, kinematics data was not synchronized with EMG data so the with-exo and without-exo conditions in the graphs were aligned by hand. In each graph, the phases of the complete lifting cycle are indicated at the bottom, along with Roman numerals I-VII to refer to these phases in the text. 

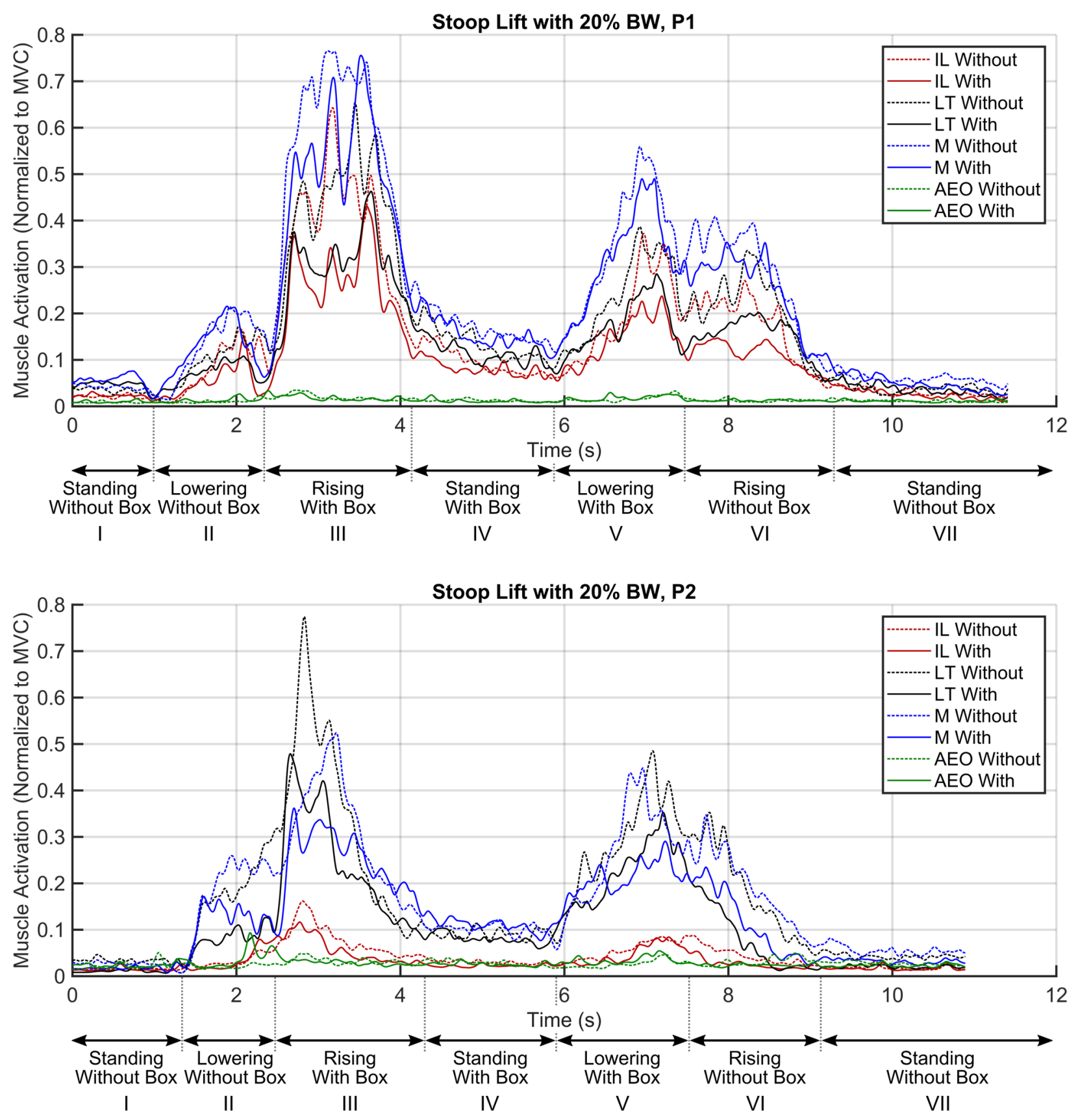

Figure A: Representative EMG signals from two individuals who performed a sequence of Stoop lifts in a pattern similar to that done during the metabolic experiments in this paper, both with and without the exoskeleton evaluated

in this paper. The muscles evaluated were the iliocostalis (IL), longissimus thoracis (LT), multifidus (M), and abdominal external obliques (AEO). Different phases of the lifting and lowering cycle are noted along the $\mathrm{x}$-axis, along with Roman numerals used to refer to them in the text. 


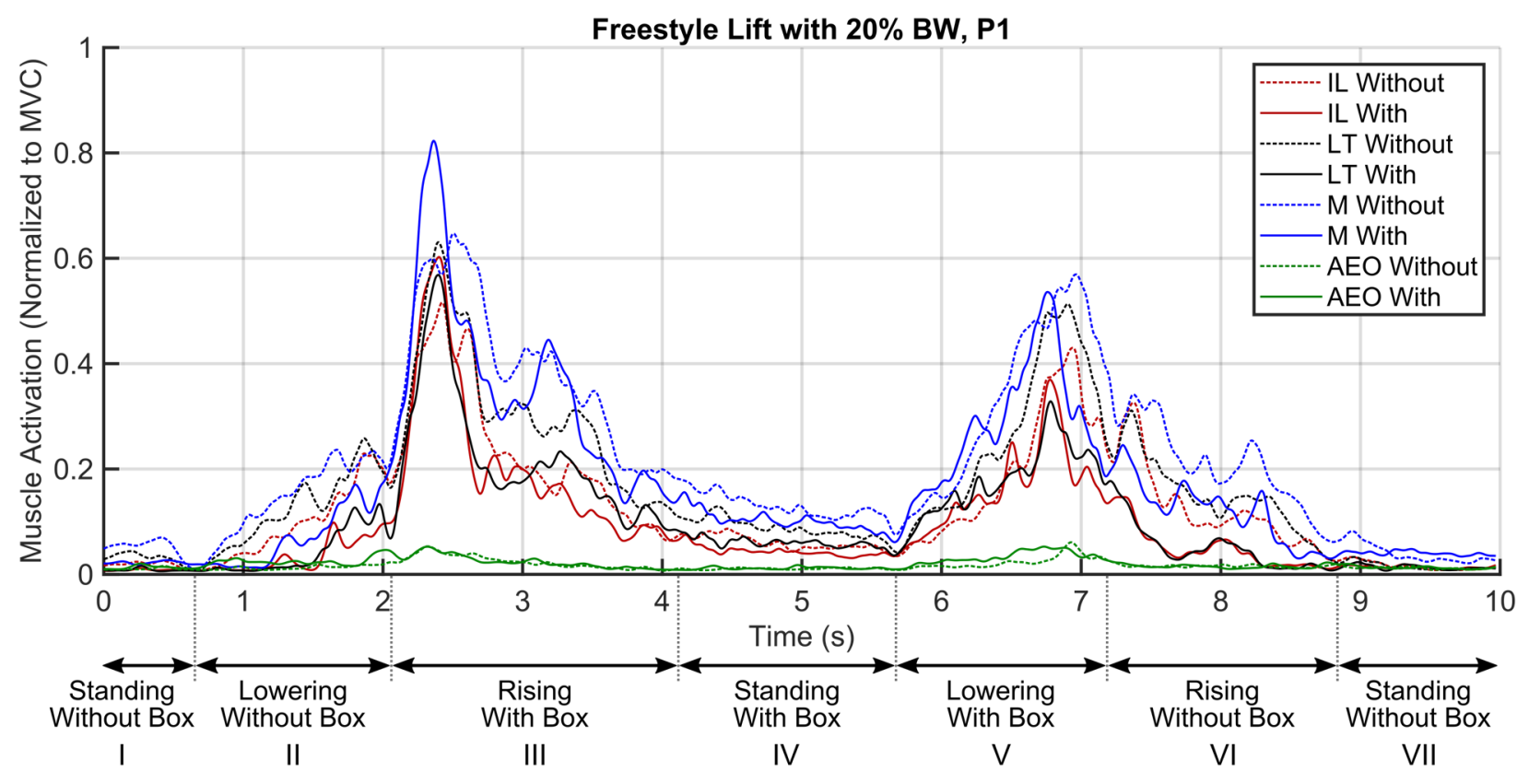

Freestyle Lift with $20 \%$ BW, P2

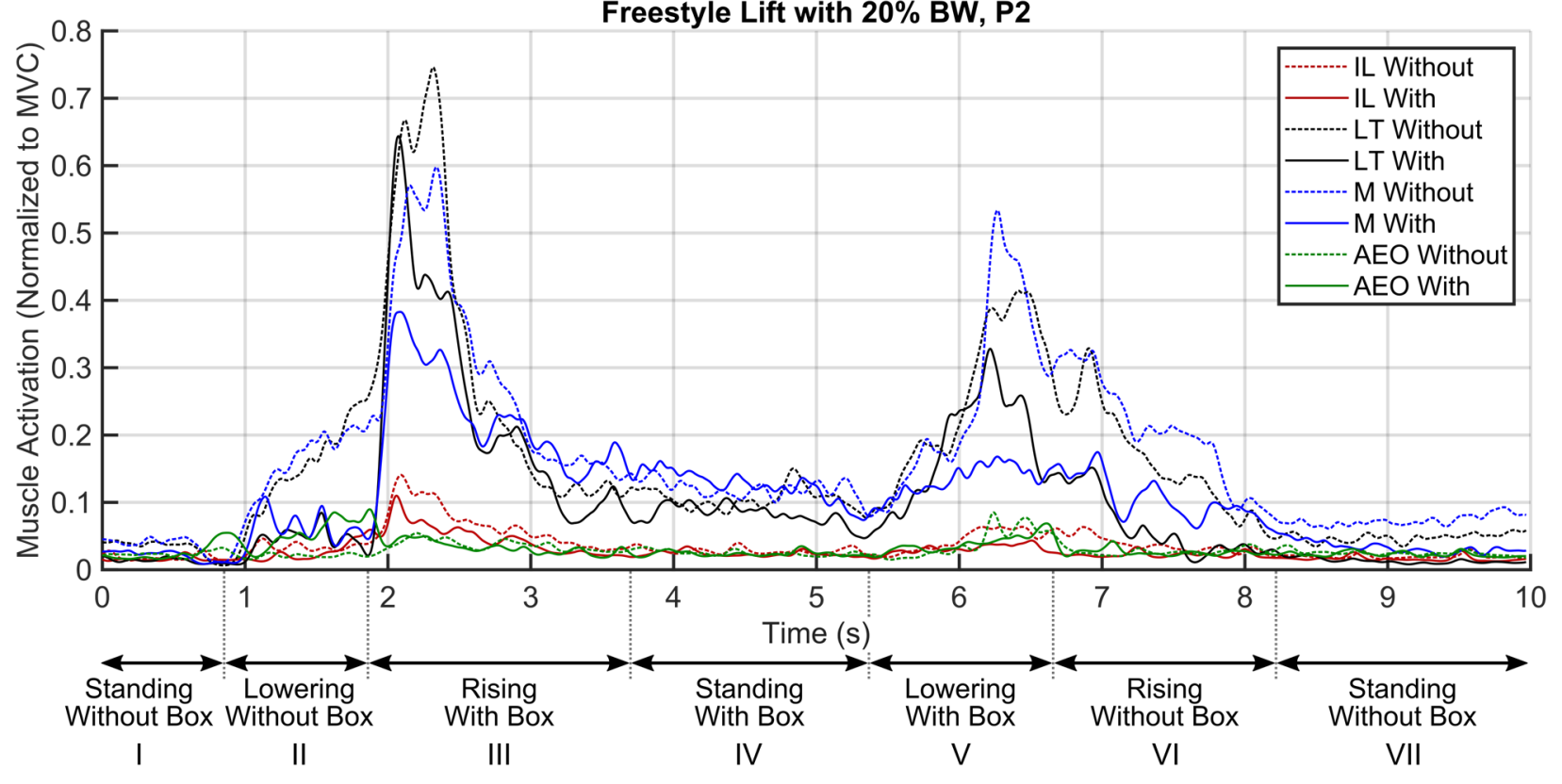

Figure B: Representative EMG signals from two individuals who performed a sequence of Freestyle lifts in a pattern similar to that done during the metabolic experiments in this paper, both with and without the exoskeleton evaluated in this paper. The muscles evaluated were the iliocostalis (IL), longissimus thoracis (LT), multifidus (M), and abdominal external obliques (AEO). Different phases of the lifting and lowering cycle are noted along the x-axis, along with Roman numerals used to refer to them in the text.

In both Figures $\mathrm{A}$ and $\mathrm{B}$, the muscle activity of both the back and stomach muscles is initially at a baseline level to maintain posture (Phase I). Just before the initiation of the first bend, the muscle activity of the back muscles dips for a moment (border between Phases I and II). Presumably, a decrease in the 
back muscle activity (while contracting the stomach muscles the same amount as before) will result in a small net torque towards the stomach, which would help accelerate the torso forward during the earliest stages of a bend.

During the first bend, in which the subject is empty-handed, the back muscle activity increases relative to standing, and moreover it increases throughout the phase as the person bends down further. This is to be expected because an inclined torso (which occurs in both Stoop and Freestyle) has an external torque due to gravity on it, which must be resisted by the back muscles. This torso angle (and the corresponding torque) increases monotonically as the person bends down, and the back muscles can also be observed to increase monotonically.

Comparing the with-exo and without-exo conditions, the back muscles are observed to decrease in magnitude substantially while wearing the exoskeleton, by more than $50 \%$ in some cases. This occurs because the back exoskeleton creates a torque pushing the torso back toward vertical, so that a smaller torque from the back muscles maintains the balance of torques around the torso. During the lowering period, participant P1 shows almost no change in stomach muscle activity, but with P2 a small increase can be observed toward the end of the lowering period (when the person's torso is bent forward the furthest and the exoskeleton's torque is highest). The stomach muscle activity in P2 could be to stabilize the torso, since there is very little back muscle activity at this point, or potentially could act to pull against the exoskeleton if the exoskeleton's torque is larger than that required to support the torso at this posture. However, note that the stomach muscles are not very active at all, with magnitudes less than $0.1 \mathrm{MVC}$, as compared to the longissimus thoracis and multifidus which are between 0.1-0.2 MVC in this phase of the lifting cycle. We note that the small amount of stomach muscle activity in our experiment agrees with prior studies of stomach muscle activity during lifting, which found that both the rectus abdominis and abdominal external obliques have very small magnitudes $(0.03-0.11 \mathrm{MVC})$ during repetitive lifting $(60,79)$. In our original study (6), the change in stomach muscle activity due to the exoskeleton over the entire lifting cycle was not found to be statistically significant. If the decrease in back muscle activity was caused by the stomach muscles pulling against the exoskeleton, we would expect the change in stomach muscles to be very apparent; however, this was not observed.

The beginning of the first rising up period (Phase III) corresponds to a slight dip then sharp increase in the back muscle activity. During this phase, too, the exoskeleton reduces the back muscle activity, by roughly the same amount (in terms of the fraction of MVC) as during the lowering phase. This is to be expected since the torque created by the exoskeleton (at each torso angle) is nearly the same during both lowering and lifting. However, this is a smaller percentage of the overall muscle activity because the peak muscle activity is much higher during this period of rising up (more than $0.6 \mathrm{MVC}$ for some muscles) than during the initial lowering (note that the person is lifting a $20 \% \mathrm{BW}$ box, which increases the torque requirements around the torso). Interestingly, the stomach muscles contract slightly at the beginning of the rising up period. This may be to help stabilize the torso or to increase the intra-abdominal pressure, thereby creating some torque to support the back. After the beginning of the lift, though, the stomach muscles have very little activity.

During the Standing with Box period (Phase IV), the muscles in the back slowly decrease in magnitude. Overall, the back muscles are contracted more than during the initial Standing period (Phase I). This is because person must hold the box in front of them; this creates a small torque pulling their torso forward, which must be resisted by the back muscles. 
When the person lowers the box again (Phase V), there is a slight dip in muscle activity just prior to the beginning of the lower, similar to the dip in back muscle activity before the lower in Phase II. During the lower, the back muscle activity again increases relative to standing, and again increases throughout the phase as the person bends down further, similar to the lower in Phase II. Also, throughout the lowering phase the stomach muscles increase in activity slightly, though the peak remains under 0.1 MVC. Again, this may be to increase the body's stability or to create some torque through intra-abdominal pressure. The exoskeleton does not appear to have any impact on the stomach muscle activity during this phase; indeed, during Freestyle lifting participant $\mathrm{P} 1$ has a slight increase in AEO activity but participant $\mathrm{P} 2$ has a decrease in AEO activity.

The border between lowering the box and rising up again without the box is observed to have a slight dip in the back EMG signals, similar to the dip between lowering without the box and lifting the box. Subsequent to this dip, the back muscles decrease in activation as the person stands up again and the external gravitational torque on their torso decreases. As with all of the other lowering and lifting phases, the back exoskeleton causes a decrease in muscle activity.

To further understand the effect of a back support exoskeleton on the torques on the torso and the activity of the stomach and back muscles, we present a very simplified view of a Stoop lift in Figure C. In the diagram, we show cartoons of the torques on the torso due to gravity, the muscles, and a possible exoskeleton, and we discuss how the exoskeleton's torque affects the back and stomach muscle activity. 


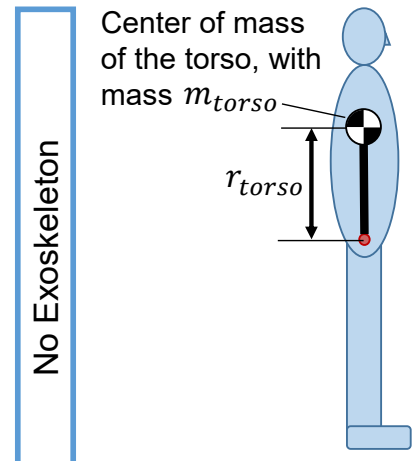

(a)

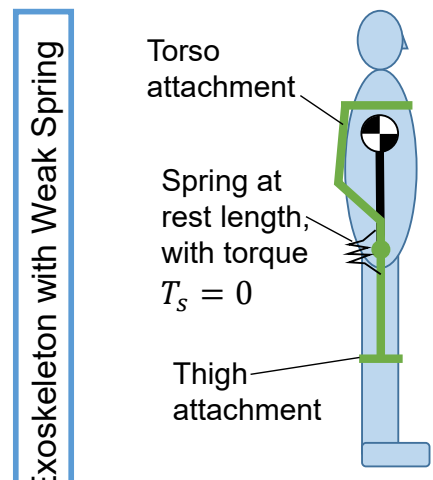

(d)

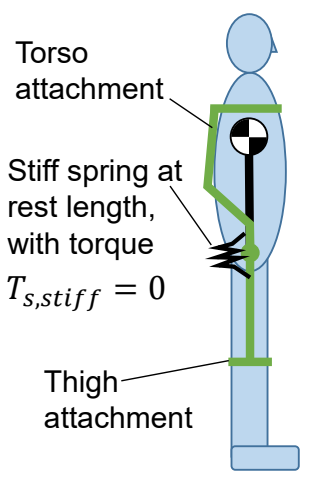

(g)

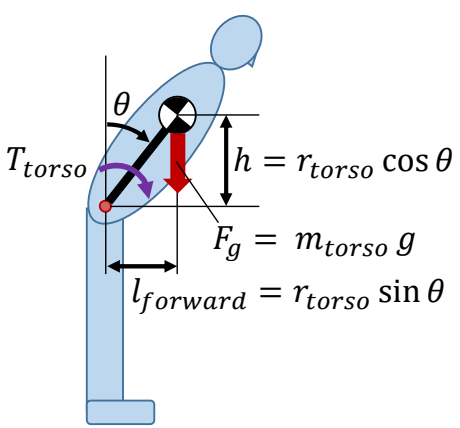

(b)

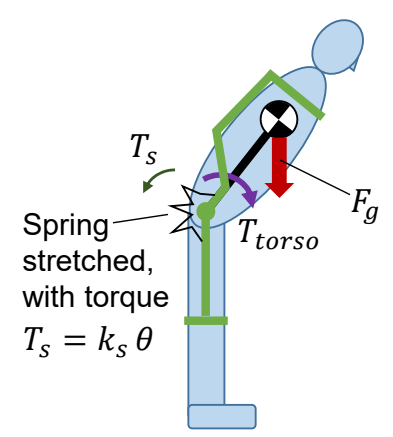

(e)

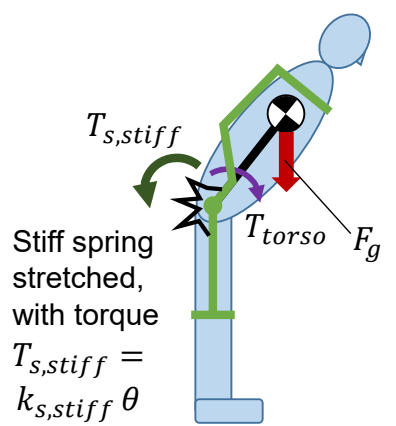

(h)

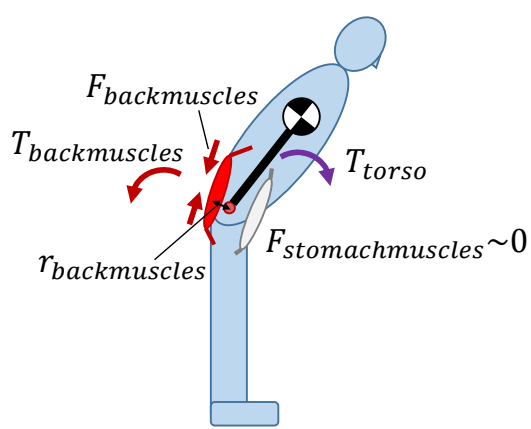

(c)

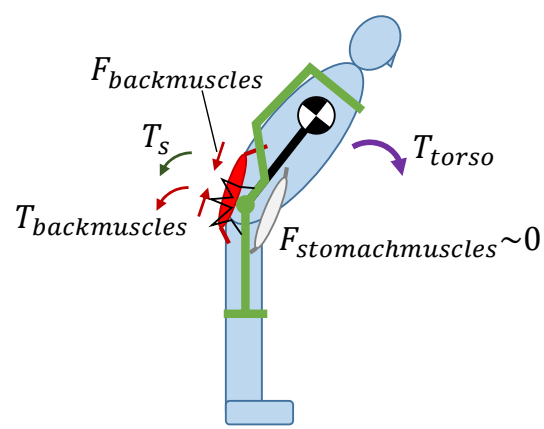

(f)

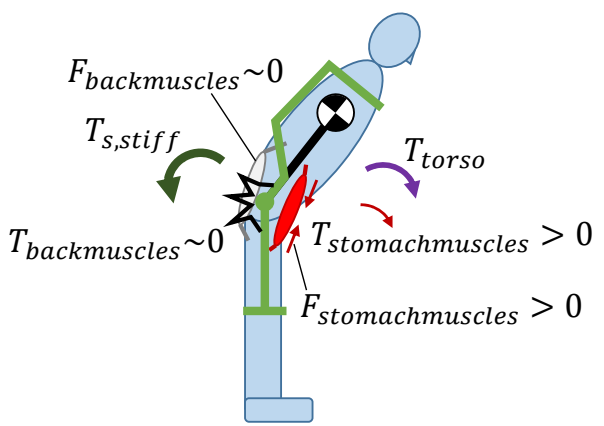

(i)

Figure C: Diagrams of a person doing a very simplified Stoop lift. The first row shows the forces on the torso and the rough activity of the back and stomach muscles during quasi-static lowering without an exoskeleton. The second row shows how those forces and muscle activity are altered with the addition of a weak or moderate-strength back support exoskeleton that provides a torque less than the torque required to support the torque on the torso due to gravity. In order for the sum of torques about the hip to remain close to zero, the back muscles reduce their forces and the stomach muscles remain largely inactive. The third row shows the forces and approximate muscle activity if a person wears a back support exoskeleton with a stiff spring that provides more torque than that required to support the torso. In this case, the back muscles reduce their activity to be close to zero and the stomach muscles must contract to achieve force balancing. In each part of the diagram, arrows for torques were drawn with different thicknesses to indicate different magnitudes (thinner lines correspond to smaller torques). 
In our example stoop lift, the person's legs remain vertical and they bend their torso forward about their hips (Figure C(a)). The torso (including head and arms) is assumed to have a total mass $m_{\text {torso }}$ which is centered at a distance $r_{\text {torso }}$ above the hip joint. When the person is standing vertically as drawn, their torso has an initial potential energy of

$$
P E_{0}=m_{\text {torso }} g r_{\text {torso }}
$$

relative to the height of the hip.

We next consider the forces on the body when the person leans forward and remains in a bent posture in static equilibrium (Figure $\mathrm{C}(\mathrm{b})$ ). Gravity always pulls down on the person's torso mass with a force $F_{g}=m_{\text {torso }} g$. When a person bends forward by an angle $\theta$, the person's torso and consequently this force are in front of their hip by a distance $l_{\text {forward }}=r_{\text {torso }} \sin \theta$. This horizontal offset of the gravitational force vector causes a torque around the hip:

$$
T_{\text {torso }}=l_{\text {forward }} F_{g}=r_{\text {torso }} \sin \theta m_{\text {torso }} g \text {. }
$$

At the position drawn, the torso mass has a potential energy relative to the hip of

$$
P E=m_{\text {torso }} g h=m_{\text {torso }} g r_{\text {torso }} \cos \theta \text {. }
$$

At this posture, we now consider the activity of the back and stomach muscles (Figure $\mathrm{C}(\mathrm{c})$ ). The back muscles are located posterior to the hip joint by a distance $r_{\text {backmuscles }}$, and create a force $F_{\text {backmuscles }}$ when they contract. This force and displacement combine to create a counterclockwise torque about the hip, $T_{\text {backmuscles }}=r_{\text {backmuscles }} F_{\text {backmuscles }}$. For the torso to remain in static equilibrium, the counterclockwise torque from the back muscles contracting must be equal in magnitude to the torque from gravity on the torso, which is pulling in the clockwise direction:

$$
\begin{gathered}
\sum M_{\text {hip }}=0: T_{\text {torso }}-T_{\text {backmuscles }}=0 \Rightarrow T_{\text {torso }}=T_{\text {backmuscles }} . \\
\text { In our simple model, this means that the back muscles have a force } \\
F_{\text {backmuscles }}=T_{\text {backmuscles }} / r_{\text {backmuscles. }} .
\end{gathered}
$$

Note that the stomach muscles do not need to contract here (shown in gray in Figure $\mathrm{C}(\mathrm{c})$ ) since they are pulling in the same direction as $T_{\text {torso }}$; if they did contract, the back muscles would have to increase their force in order to maintain static equilibrium around the hip joint. This is in accordance with the studies showing that the stomach muscles are not very active during lifting $(60,79)$.

Finally, if the person bends $90^{\circ}$ at the waist, the torso mass now has a potential energy of $P E=0$ relative to the hip, since it is at the same height as the hip. In this case, all of the potential energy that the person's torso had when it was perfectly upright has been lost (turned into heat).

We now present a very simplified model of a back exoskeleton, illustrated in the second row of Figure C. The exoskeleton has a hinge collocated with the hip joint, is secured to the body at the torso, thighs, and pelvis (not shown in the Figure), and creates torque between the torso segment and leg segment via a torsional spring located at the hip. When the person is standing vertically $(\theta=0)$, as in Figure $\mathrm{C}(\mathrm{d})$, the spring is at its rest length, creates no torque $\left(T_{S}=0\right)$, and has no stored potential energy $\left(P E_{S}=0\right)$.

If the person then bends forward at the waist by an angle $\theta$, the spring in the exoskeleton extends, creating a torque $T_{s}=k_{s} \theta$ that is in the counterclockwise direction, opposing the torso torque $T_{\text {torso }}$. This is shown in Figure $\mathrm{C}(\mathrm{e})$. There, $T_{s}$ is drawn with a smaller, thinner line than $T_{\text {torso }}$; this is because its magnitude is assumed to be smaller than $T_{\text {torso }}: T_{s}<T_{\text {torso }} . T_{s}$ being smaller than $T_{\text {torso }}$ is true for all current passive back exoskeletons to our knowledge. 
At the position drawn, the torso mass has a potential energy of $P E=m_{\text {torso }} g r_{\text {torso }} \cos \theta$ as before. Now, the exoskeleton spring has a potential energy of $P E_{s}=1 / 2 k_{s} \theta^{2}$. Its energy came from the loss of potential energy of the torso: some of the initial potential energy is transferred to potential energy in the spring. This is similar to many spring-powered passive gravity compensation mechanisms for a mass on an arm at some radius from a joint, where a single spring (possibly in conjunction with a non-circular pulley) can perfectly offset the weight of the mass $(26,66)$. These mechanisms take great care to create a torque with the spring and linkage that perfectly matches the torque due to gravity on the mass, and as a result the compensated mass appears "weightless" in all possible arm angles. With an exoskeleton, we do not have a very precise equation for the torque required to support the torso, so current exoskeletons provide a weaker torque than that required to support the torso. This strategy also allows the exoskeletons to fit on individuals with a range of torso masses. We note that the torque provided by an exoskeleton is a function of the torso angle, similar to the gravity compensation system: with the torso inclined forward only slightly, the torque is smaller than the torque when the torso is nearly horizontal.

Looking now at the back and stomach muscle activity in Figure $\mathrm{C}(\mathrm{f})$, we observe that since the exoskeleton torque is counteracting $T_{\text {torso }}$ but has a smaller magnitude than $T_{\text {torso }}$, the back muscles must still engage to create a counterclockwise torque about the hip. The total torque of exoskeleton plus body muscles must equal the $T_{\text {torso }}$ for static equilibrium:

$$
\sum M_{\text {hip }}=0: T_{\text {torso }}-T_{\text {backmuscles }}-T_{s}=0 \Rightarrow T_{\text {torso }}=T_{\text {backmuscles }}+T_{s} .
$$

Now, the forces on the back muscles are somewhat decreased:

$$
\begin{gathered}
T_{\text {backmuscles }}=T_{\text {torso }}-T_{s} \\
F_{\text {backmuscles }}=\left(T_{\text {torso }}-T_{s}\right) / r_{\text {backmuscles }}
\end{gathered}
$$

This is smaller than the force without the exoskeleton, but is still positive (i.e., the back muscles still need to contract) since $T_{\text {torso }}>T_{s}$. Indeed, this is what is observed in Figures A and B. There, the back muscles decrease in activation while the person is lowering (Phase II). Even though the muscles are extending eccentrically, they still require some energy to produce force. Thus, metabolic savings can occur if the back muscles decrease in force. We also note that the stomach muscles still do not need to contract in this circumstance (shown in gray in the picture), since the exoskeleton torque $T_{S}$ is smaller than the torque required to support the torso. If the stomach muscles contracted, then the back muscles would need to increase in force to maintain static equilibrium. This co-contraction may be useful to increase the torso's stability, but does not seem occur to a significant extent in practice (Figures A and B).

Finally, if the person bends $90^{\circ}$ at the waist, the torso mass again has a potential energy of $P E=0$ relative to the hip. The exoskeleton spring now has $P E_{S}=1 / 2 k_{S} \theta^{2}$ with $\theta=90^{\circ}$. This stored energy is less than that lost by the torso moving downward, i.e. $P E_{S}<P E_{0}$, since the exoskeleton had a comparatively weak spring.

We next consider what happens if there is an extremely stiff exoskeleton, illustrated in the third row of Figure C. This exoskeleton has a spring constant of $k_{s, s t i f f} \gg k_{s}$. As before, when the person is standing upright the spring is at its rest length, the exoskeleton creates no torque $\left(T_{s, s t i f f}=0\right)$ and has no stored potential energy $\left(P E_{s, s t i f f}=0\right)$.

Again, if the person bends forward (Figure $\mathrm{C}(\mathrm{h})$ ), the spring in the exoskeleton extends, creating a torque $T_{s, \text { stiff }}=k_{s, \text { stiff }} \theta$ that opposes the torso torque $T_{\text {torso }}$. Note that $T_{s, \text { stiff }}$ is drawn with a larger, 
thicker line than $T_{\text {torso }}$; this is because now, with the stiff spring, its magnitude is larger than $T_{\text {torso }}$ : $T_{s, \text { stiff }}>T_{\text {torso }}$. No current passive back exoskeletons have a spring this stiff.

At the position drawn, the torso mass has a potential energy of $P E=m_{\text {torso }} g r_{\text {torso }} \cos \theta$ as before. Now, the exoskeleton spring has a potential energy of $P E_{s, \text { stiff }}=1 / 2 k_{s, s t i f f} \theta^{2}$. Since the spring is stiff, its stored energy is now greater than that lost by the torso:

$$
P E_{s, \text { stiff }}>\left(P E_{0}-P E\right) \text {. }
$$

Since the exoskeleton torque now has a larger magnitude than $T_{\text {torso }}$, the stomach muscles must now contract to create static equilibrium. The total torque of exoskeleton plus muscles must still equal $T_{\text {torso }}$ for static equilibrium. But now, the stomach muscles create a torque in the clockwise direction so we get:

$$
\begin{gathered}
\sum M_{\text {hip }}=0: T_{\text {torso }}+T_{\text {stomachmuscles }}-T_{s, \text { stiff }}=0 \\
\Rightarrow T_{s, \text { stiff }}=T_{\text {torso }}+T_{\text {stomachmuscles }} .
\end{gathered}
$$

Since the exoskeleton torque with the stiff spring is more than that required to support the torso, the back muscles do not need to contract. If they did, they would require the stomach muscles to contract even more.

Finally, if the person bends $90^{\circ}$ at the waist, the torso mass again has a potential energy of $P E=0$ relative to the hip. The exoskeleton spring now has a stored potential energy of $P E_{s, \text { stiff }}=1 / 2 k_{s, s t i f f} \theta^{2}$ with $\theta=90^{\circ}$. With the stiff spring, the stored energy is now more than that lost by the torso moving downward, i.e. $P E_{s, s t i f f}>P E_{0}$. The extra energy in this case was contributed by the stomach muscles contracting.

Of course, we have made a large number of simplifying assumptions in this model. First, thus far we have considered everything to be quasi-static, i.e. that the system was moving slowly enough that the dynamics of the system could be ignored. This quasi-static assumption is indeed generally correct for lifting: people move relatively slowly, and the kinetic energy of the system does not play a large part in the total system energy, as discussed at the end of Modeling Part A. Overall, the torso does have inertia and there is damping due to the structures around the waist. If a person had no muscle activity in their back or stomach and their torso was left to fall over under the influence of gravity, it would move with some characteristic velocity versus time. If the person were to bend more slowly than this, then the back muscles must be used to arrest the fall, and lower the person's torso gradually by creating a counterclockwise torque. This quasi-static motion is what happens in practice when people bend down to pick something up, and what was modeled in the preceding pages of this discussion. If the person were to bend more quickly than this, then the stomach muscles must be used to accelerate the torso toward the floor more quickly than it would fall naturally, with a clockwise torque.

While overall the lifting motion is quasi-static, we do observe some small changes in muscle activity (even without the exoskeleton) that probably contribute to the torso accelerating forward before each lower (the dips in back muscle activity before each lower) and to either stabilization or acceleration at the deepest portion of the bend (the small increases in stomach muscle activity there). However, these are quite small compared to the overall activity of the back muscles (and hip extensors such as the biceps femoris) that create torques on the torso, and as such do not present significant errors in our model.

Additionally, our simplified model is only for a stoop lift style and assumes that the lower body essentially is held in place vertically. In practice, for quasi-static motions a person must control the location 
of their whole-body center of mass so that it remains within the support polygon made by their feet; this corresponds to moving forward and backward a small amount. Similarly, for freestyle and squat lifting the knees bend a substantial amount during lifting. With an exoskeleton, the torque is a function of the angle from the shoulders to the hips to the knees, not the torso angle itself. Thus, an exoskeleton bends more during squat and freestyle lifts than during stoop lifts, and has a consequently higher torque and stored energy (19). However, our overall analysis of the torques on the torso is the same in all cases. The extra torque from the exoskeleton during Freestyle lifting as compared to Stoop lifting is what leads to greater reductions in back muscle activity for Freestyle lifting as compared to Stoop lifting (see (6) for a study with the exoskeleton evaluated in this paper; this can also be observed in Figures A and B).

As a final note, since the exoskeleton is using the torso's potential energy as the energy source for its stored energy, the exoskeleton only works properly if the person's torso is initially upright and they lean forward. This is what occurs during lifting in practice, when a person bends forward to reach an object. For comparison, if a person lies down on the floor and then does a sit-up while wearing an exoskeleton, the person will have to contract their stomach muscles more than they normally would and fight against the exoskeleton. This is because the torque on the torso due to gravity is pulling their torso toward the ground, which in this case is also how the exoskeleton is pulling on the torso (the exoskeleton tries to pull the torso to be in line with the thighs).

\section{Appendix C}

In studies (22) and (30), participants either lifted or lowered a box, and the box was returned to its original height by other means (a powered mechanism or another person, respectively). While both experiments used small numbers of subjects ( $\mathrm{N}=6$ in (22) and $\mathrm{N}=3$ males in (30)), they can provide some insight into the ratio $R_{B o x}=\frac{K_{B o x, l i f t}}{K_{B o x, l o w e r}}$. The study by (22) used freestyle lifting and resulted in best-fit values of $K_{B o d y}=6.06, K_{B o x, l i f t}=7.02$, and $K_{B o x, \text { lower }}=4.17$. The sum of $K_{B o x, \text { lift }}$ and $K_{B o x, l o w e r}$ is $K_{B o x}=$ 11.19 , and their ratio $R_{B o x}$ is 1.68 . The study by (30) used both stoop and squat lifting. Stoop lifts had bestfit values of $K_{B o d y}=2.75, K_{B o x, \text { lift }}=9.27, K_{B o x \text {, lower }}=2.45$; this corresponds to $K_{B o x}=11.73$ and $R_{B o x}$ $=3.78$. Squat lifts had best-fit values of $K_{B o d y}=3.12, K_{B o x, \text { lift }}=9.79, K_{B o x, \text { lower }}=1.85$; this corresponds to $K_{B o x}=11.64$ and $R_{B o x}=5.29$. These results and the $K$-values from the other studies mentioned in this paper are in Table A for ease of comparison, and plots of the predicted versus experimental data are in Figures D, E, and F.

The values of $K_{\text {Body }}$ in (22) and (30) differ somewhat from the values in the previous experiments. This is likely due to the smaller sample sizes in those experiments, or possibly is due to different postures used to lift and lower the load. In (22), a barbell was used to hold the weight, and participants paused at the bottom of the lift for $\sim 0.5$ seconds. The metabolic cost of lifting in that study is much greater than that in any of the other studies referenced in this paper, for unknown reasons. In (30), the participants also pushed the object onto a table, which was not considered in the model, but would be a roughly constant value that would be included in $K_{\text {Body }}$. However, in both experiments the values of $K_{B o x}$ are fairly similar to the other experiments discussed in this paper ( 11.2-11.7, as compared to 10.7-14.3). Unfortunately, the estimated ratios $R_{B o x}$ vary substantially between the experiments, from 1.68 to 5.29 . It appears that the ratio is definitely greater than one, however. Further experimentation with a larger number of subjects will give more insight into this value. 
Table A: Summary of the $K$-values for different experiments as well as the number of subjects and lifting frequencies used in each experiment.

\begin{tabular}{|c|c|c|c|c|c|}
\hline & K-value & $\begin{array}{c}\text { Hagen (1994), } \\
\text { Welbergen (1991) }\end{array}$ & Current study & Garg (1976) & De Looze (1994) \\
\hline No. Subj. & & $10 \mathrm{M}, 9 \mathrm{M}$ & $15(13 \mathrm{M} \&$ 2F) & $3 \mathrm{M}$ & $6 \mathrm{M}$ \\
\hline Lift Freq. & & 10,10 & $12^{*}$ & $4,8,12$ & 12.76 \\
\hline \multirow{3}{*}{ Stoop } & $K_{\text {Body }}$ & 3.85 & & 2.75 & \\
\cline { 2 - 6 } & $\begin{array}{c}K_{\text {Box }} \\
\text { (Lift, Lower) }\end{array}$ & 10.67 & & $\begin{array}{c}11.73 \\
(9.27,2.45)\end{array}$ & \\
\hline \multirow{3}{*}{ Squat } & $K_{\text {Body }}$ & 4.48 & & 3.12 & \\
\cline { 2 - 6 } & $\begin{array}{c}K_{\text {Box }} \\
(\text { Lift, Lower) }\end{array}$ & 13.26 & & 11.64 & $(9.79,1.85)$ \\
\hline \multirow{3}{*}{ Freestyle } & $K_{\text {Body }}$ & & 4.31 & & 6.06 \\
\cline { 2 - 6 } & $\begin{array}{c}K_{\text {Box }} \\
\text { (Lift, Lower) }\end{array}$ & & 14.30 & & 11.19 \\
\hline
\end{tabular}

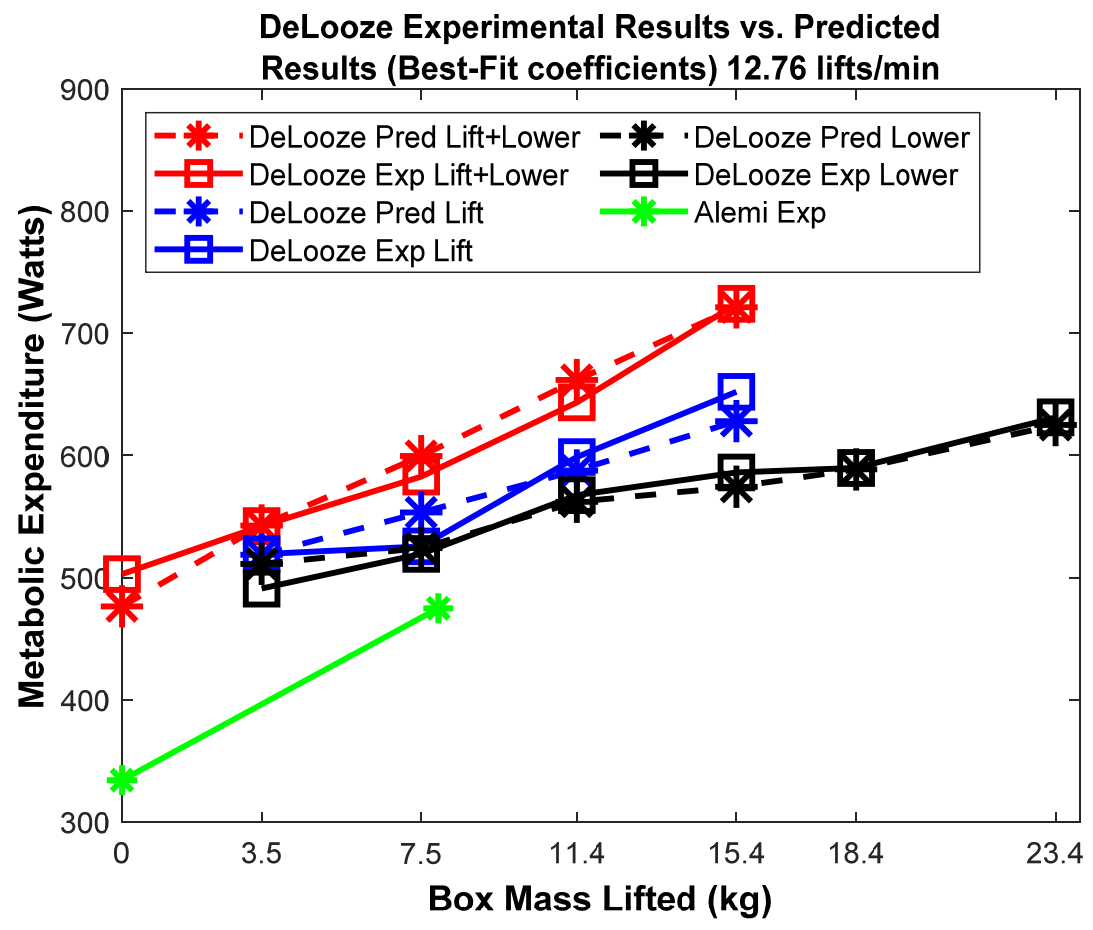

Figure D: Predicted metabolic cost vs. experimental results in (22) for freestyle lifting at $12.76 \mathrm{lifts} / \mathrm{min}(\mathrm{N}=6$ Males). The experimental data from this paper $(\mathrm{N}=15 ; 13 \mathrm{M} \& 2 \mathrm{~F})$ is plotted for comparison; the point for $20 \% \mathrm{BW}$ mass is plotted at $7.93 \mathrm{~kg}=15.85 \mathrm{~kg} / 2$ since the weighted box was only held for half of the complete lifting cycle. It is unknown why the metabolic cost of lifting in the De Looze paper is substantially higher than all of the other data mentioned in this paper. The higher metabolic cost corresponds to a larger value of $K_{\text {Body }}$. 


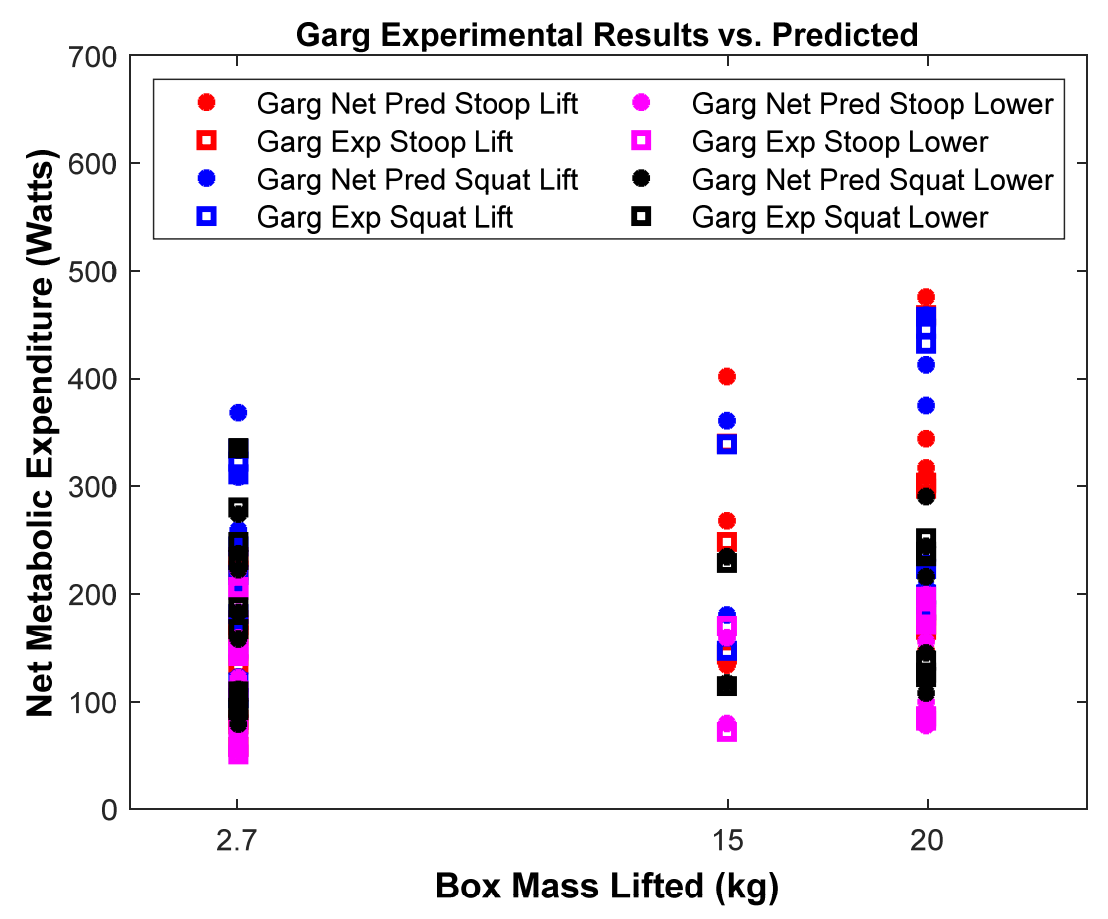

Figure E: Predicted net metabolic cost vs. experimental results in (30) for stoop and squat lifts and lowers at 4, 8 , and 12 lifts/min $(\mathrm{N}=3$ Males $)$. Note that this is the net metabolic expenditure, which does not include the metabolic cost of resting. 


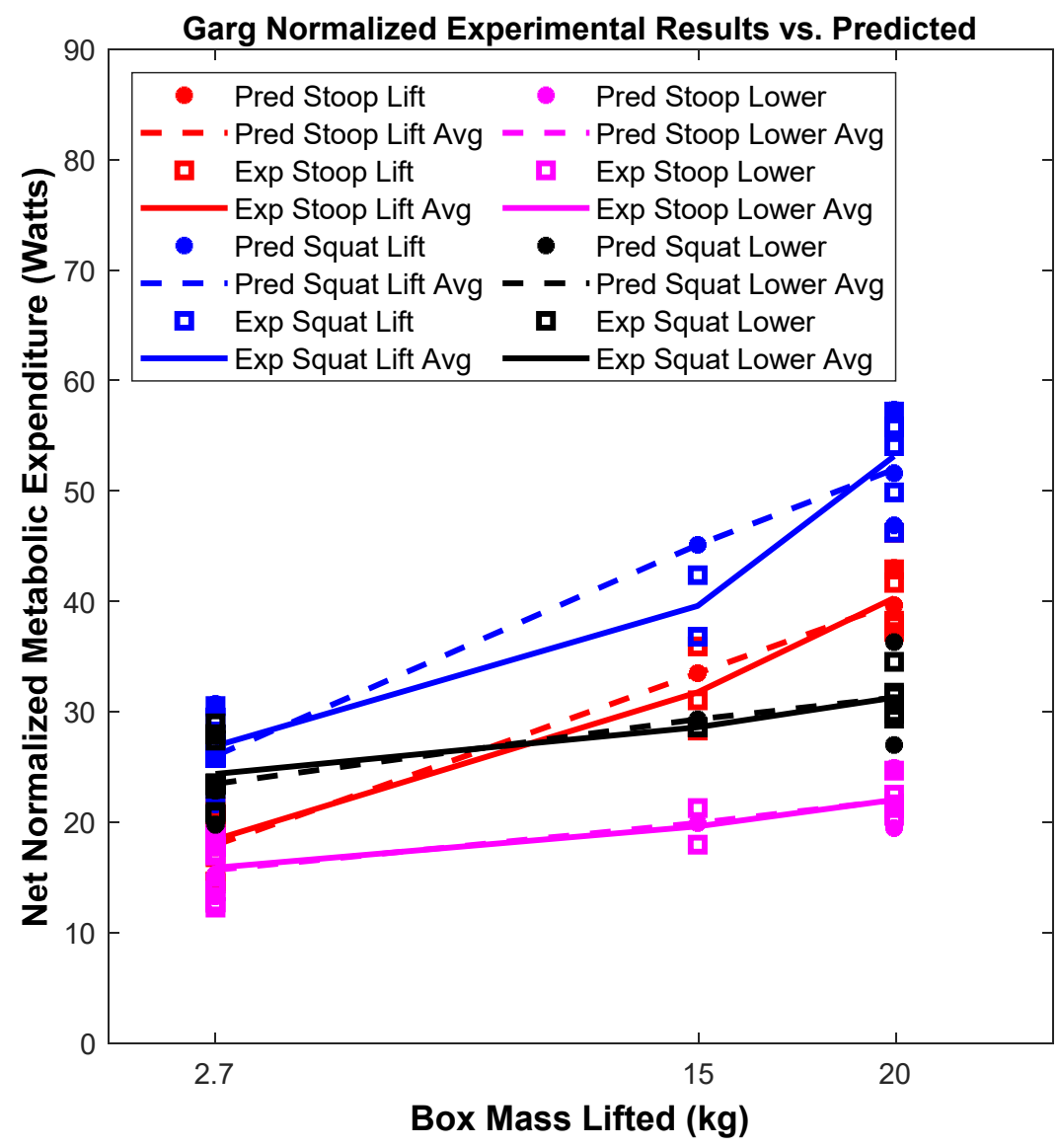

Figure F: Comparison between the normalized experimental results from (30) and the normalized predicted values ( $\mathrm{N}=3$ Males). In this plot, the net metabolic expenditure (that is, excluding resting) is normalized with respect to the lift frequency ( $L=4,8,12$ lifts per minute) so that the different lifting frequencies align. The solid (experimental) and dashed (predicted) lines pass through the average of the normalized data for each condition. 


\section{References}

1. Abbott BC, Bigland B, Ritchie JM. The physiological cost of negative work. J Physiol 117: 380-390, 1952. doi: 10.1113/jphysiol.1952.sp004755.

2. Abdoli-e M, Agnew MJ, Stevenson JM. An on-body personal lift augmentation device ( PLAD ) reduces EMG amplitude of erector spinae during lifting tasks. 21: 456-465, 2006. doi: 10.1016/j.clinbiomech.2005.12.021.

3. Abdoli-e M, Stevenson JM. The effect of on-body lift assistive device on the lumbar 3D dynamic moments and EMG during asymmetric freestyle lifting. 23: 372-380, 2008. doi: 10.1016/j.clinbiomech.2007.10.012.

4. Aberg U, Elgstrand K, Magnus P, Lindholm A. Analysts of components and prediction of energy expenditure in manual tasks. Int J Prod Res 6: 189-196, 1967. doi: 10.1080/00207546708929779.

5. Alemi MM. Biomechanical Assessment and Metabolic Evaluation of Passive Lift-Assistive Exoskeletons During Repetitive Lifting Tasks. (Unpublished Doctoral dissertation, Virginia Tech): 2019.

6. Alemi MM, Geissinger J, Simon AA, Chang SE, Asbeck AT. A passive exoskeleton reduces peak and mean EMG during symmetric and asymmetric lifting. J Electromyogr Kinesiol 47: 2534, 2019. doi: 10.1016/j.jelekin.2019.05.003.

7. Alemi MM, Madinei S, Kim S, Srinivasan D, Nussbaum MA. Effects of Two Passive BackSupport Exoskeletons on Muscle Activity, Energy Expenditure, and Subjective Assessments During Repetitive Lifting. Hum Factors J Hum Factors Ergon Soc 62: 458-474, 2020. doi: 10.1177/0018720819897669.

8. Aquilano NJ. Physiological evaluation of time standards for strenuous work as set by stopwatch time study and two predetermined motion time data system. $J$ Ind Eng XIX: 425-432, 1968.

9. Asfour SS. Energy cost prediction models for manual lifting and lowering tasks. (Doctoral Dissertation, Texas Tech University): 1980.

10. Baltrusch SJ, van Dieën JH, Bruijn SM, Koopman AS, van Bennekom CAM, Houdijk H. The effect of a passive trunk exoskeleton on metabolic costs during lifting and walking. Ergonomics 62: 903-916, 2019. doi: 10.1080/00140139.2019.1602288.

11. Baltrusch SJ, van Dieën JH, Koopman AS, Näf MB, Rodriguez-Guerrero C, Babič J, Houdijk H. SPEXOR passive spinal exoskeleton decreases metabolic cost during symmetric repetitive lifting. Eur J Appl Physiol 120: 401-412, 2020. doi: 10.1007/s00421-019-04284-6.

12. Baltrusch SJ, van Dieën JH, van Bennekom CAM, Houdijk $\mathbf{H}$. The effect of a passive trunk exoskeleton on functional performance in healthy individuals. Appl Ergon 72: 94-106, 2018. doi: 10.1016/j.apergo.2018.04.007.

13. Bell JA, Burnett A. Exercise for the Primary, Secondary and Tertiary Prevention of Low Back Pain in the Workplace: A Systematic Review. J Occup Rehabil 19: 8-24, 2009. doi: 10.1007/s10926-009-9164-5.

14. Bigos SJ, Holland J, Holland C, Webster JS, Battie M, Malmgren JA. High-quality controlled trials on preventing episodes of back problems: systematic literature review in working-age adults. Spine J 9: 147-168, 2009. doi: 10.1016/j.spinee.2008.11.001.

15. Bogue R. Exoskeletons and robotic prosthetics: A review of recent developments. Ind Rob 36: 421-427, 2009. doi: 10.1108/01439910910980141.

16. Bosch T, van Eck J, Knitel K, de Looze M. The effects of a passive exoskeleton on muscle activity, discomfort and endurance time in forward bending work. Appl Ergon 54: 212-217, 2016. doi: 10.1016/j.apergo.2015.12.003.

17. Brockway JM. Derivation of formulae used to calculate energy expenditure in man. Hum Nutr Clin Nutr 41: 463-71, 1987.

18. Burke MJ, Sarpy SA, Smith-Crowe K, Chan-Serafin S, Salvador RO, Islam G. Relative Effectiveness of Worker Safety and Health Training Methods. Am J Public Health 96: 315-324, 2006. doi: 10.2105/AJPH.2004.059840. 
19. Chang SE, Pesek T, Pote TR, Hull J, Geissinger J, Simon AA, Alemi MM, Asbeck AT. Design and preliminary evaluation of a flexible exoskeleton to assist with lifting. Wearable Technol 1, 2020. doi: 10.1017/wtc.2020.10.

20. da Costa BR, Vieira ER. Risk factors for work-related musculoskeletal disorders: a systematic review of recent longitudinal studies. .

21. Daltroy LH, Iversen MD, Larson MG, Lew R, Wright E, Ryan J, Zwerling C, Fossel AH, Liang MH. A Controlled Trial of an Educational Program to Prevent Low Back Injuries. $N$ Engl J Med 337: 322-328, 1997. doi: 10.1056/NEJM199707313370507.

22. De Looze MP, Toussaint HM, Commissaris DA, Jans MP, Sargeant AJ. Relationships between energy expenditure and positive and negative mechanical work in repetitive lifting and lowering. J Appl Physiol 77: 420-426, 1994. doi: 10.1152/jappl.1994.77.1.420.

23. de Looze MP, Toussaint HM, Nibbelke RJ, Eelderink HA. Effects on efficiency in repetitive lifting of load and frequency combinations at a constant total power output. Eur J Appl Physiol Occup Physiol 65: 469-474, 1992. doi: 10.1007/BF00243516.

24. Duffield R, Dawson B, Pinnington HC, Wong P. Accuracy and reliability of a Cosmed K4b 2 portable gas analysis system. J Sci Med Sport 7: 11-22, 2004. doi: 10.1016/S14402440(04)80039-2.

25. Dutta SP, Taboun S. Developing norms for manual carrying tasks using mechanical efficiency as the optimization criterion. Ergonomics 32: 919-943, 1989. doi: 10.1080/00140138908966855.

26. Endo G, Yamada H, Yajima A, Ogata M, Hirose S. A passive weight compensation mechanism with a non-circular pulley and a spring. Proc - IEEE Int Conf Robot Autom 3: 3843-3848, 2010. doi: 10.1109/ROBOT.2010.5509797.

27. Fanello S, Jousset N, Roquelaure Y, Chotard-Frampas V, Delbos V. Evaluation of a training program for the prevention of lower back pain among hospital employees. Nurs Heal Sci 4: 51-54, 2002. doi: 10.1046/j.1442-2018.2002.00098.x.

28. Frost DM, Abdoli-E M, Stevenson JM. PLAD (personal lift assistive device) stiffness affects the lumbar flexion/extension moment and the posterior chain EMG during symmetrical lifting tasks. $J$ Electromyogr Kinesiol 19: e403-e412, 2009. doi: 10.1016/j.jelekin.2008.12.002.

29. Gaesser GA, Brooks GA. Muscular efficiency during steady-rate exercise: effects of speed and work rate. J Appl Physiol 38: 1132-1139, 1975. doi: 10.1152/jappl.1975.38.6.1132.

30. Garg A. A METABOLIC RATE PREDICTION MODEL FOR MANUAL MATERIALS HANDLING JOBS. The University of Michigan: 1976.

31. Garg A, Ayoub MM. What criteria exist for determining how much load can be lifted safely? Hum Factors 22: 475-486, 1980. doi: 10.1177/001872088002200408.

32. Garg A, Chaffin DB, Herrin GD. Prediction of metabolic rates for manual materials handling jobs. Am Ind Hyg Assoc J 39, 1978.

33. Garg A, Herrin GD. Stoop or squat: a biomechanical and metabolic evaluation. AIIE Trans 11: 293-302, 1979.

34. Gatty CM, Turner M, Buitendorp DJ, Batman H. The effectiveness of back pain and injury prevention programs in the workplace. Work 20: 257-66, 2003.

35. Gebhardt WA. Effectiveness of training to prevent job-related back pain: A meta-analysis. $\mathrm{Br} J$ Clin Psychol 33: 571-574, 1994. doi: 10.1111/j.2044-8260.1994.tb01153.x.

36. Givoni B, Goldman RF. Predicting metabolic energy cost. J Appl Physiol 30: 429-433, 1971. doi: 10.1152/jappl.1971.30.3.429.

37. Godwin AA, Stevenson JM, Agnew MJ, Twiddy AL, Abdoli-Eramaki M, Lotz CA. Testing the efficacy of an ergonomic lifting aid at diminishing muscular fatigue in women over a prolonged period of lifting. Int J Ind Ergon 39: 121-126, 2009. doi: 10.1016/j.ergon.2008.05.008.

38. Gordon CC, Churchill T, Clauser CE, Bradtmiller B, McConville JT, Tebbetts I, Walker RA. 1988 Anthropometric survey of US army personnel: methods and summary statistics. Technical Report Natick/TR-89/044. Natick, Massachusetts: 1989.

39. Griffith LE, Shannon HS, Wells RP, Walter SD, Cole DC, Côté P, Frank J, Hogg-Johnson S, 
Langlois LE. Individual Participant Data Meta-Analysis of Mechanical Workplace Risk Factors and Low Back Pain. Am J Public Health 102: 309-318, 2012. doi: 10.2105/AJPH.2011.300343.

40. Guo HR, Tanaka S, Halperin WE, Cameron LL. Back pain prevalence in US industry and estimates of lost workdays. Am J Public Health 89: 1029-1035, 1999. doi:

10.2105/AJPH.89.7.1029.

41. Hagen KB, Harms-Ringdahl K, Hallén J. Influence of lifting technique on perceptual and cardiovascular responses to submaximal repetitive lifting. Eur J Appl Physiol Occup Physiol 68: 477-482, 1994. doi: 10.1007/BF00599516.

42. Hake M, Michael ED. The physiological costs of box lifting. J Hum Ergol (Tokyo) 6: 167-78, 1977.

43. Hausswirth C, Bigard A, Le Chevalier J. The Cosmed K4 Telemetry System as an Accurate Device for Oxygen Uptake Measurements during Exercise. Int J Sports Med 28: 449-453, 2007. doi: 10.1055/s-2007-972662.

44. Hody S, Croisier J-L, Bury T, Rogister B, Leprince P. Eccentric Muscle Contractions: Risks and Benefits. Front Physiol 10, 2019. doi: 10.3389/fphys.2019.00536.

45. Jiang BCC. A manual materials handling study of bag lifting. (Master Dissertation, Texas Tech University): 1981.

46. Kamon E. Negative and positive work in climbing a laddermill. $J$ Appl Physiol 29: 1-5, 1970. doi: 10.1152/jappl.1970.29.1.1.

47. Kelsey JL, Githens PB, White AA, Holford TR, Walter SD, O'Connor T, Ostfeld AM, Weil U, Southwick WO, Calogero JA. An epidemiologic study of lifting and twisting on the job and risk for acute prolapsed lumbar intervertebral disc. J Orthop Res 2: 61-66, 1984. doi: 10.1002/jor.1100020110.

48. Kim S, Nussbaum MA, Mokhlespour Esfahani MI, Alemi MM, Alabdulkarim S, Rashedi E. Assessing the influence of a passive, upper extremity exoskeletal vest for tasks requiring arm elevation: Part I - "Expected" effects on discomfort, shoulder muscle activity, and work task performance. Appl Ergon 70, 2018. doi: 10.1016/j.apergo.2018.02.025.

49. Kim S, Nussbaum MA, Mokhlespour Esfahani MI, Alemi MM, Jia B, Rashedi E. Assessing the influence of a passive, upper extremity exoskeletal vest for tasks requiring arm elevation: Part II - "Unexpected" effects on shoulder motion, balance, and spine loading. Appl Ergon 70, 2018. doi: 10.1016/j.apergo.2018.02.024.

50. Koopman AS, Kingma I, Faber GS, de Looze MP, van Dieën JH. Effects of a passive exoskeleton on the mechanical loading of the low back in static holding tasks. J Biomech 83: 97 103, 2019. doi: 10.1016/j.jbiomech.2018.11.033.

51. Kumar S. The physiological cost of three different methods of lifting in sagittal and lateral planes. Ergonomics 27: 425-433, 1984. doi: 10.1080/00140138408963506.

52. Laevo. Laevo. 2017.

53. Lahad A. The Effectiveness of Four Interventions for the Prevention of Low Back Pain. JAMA J Am Med Assoc 272: 1286, 1994. doi: 10.1001/jama.1994.03520160070046.

54. Lamers EP, Yang AJ, Zelik KE. Feasibility of a Biomechanically-Assistive Garment to Reduce Low Back Loading During Leaning and Lifting. IEEE Trans Biomed Eng 65: 1674-1680, 2018. doi: 10.1109/TBME.2017.2761455.

55. Lavender SA, Ko P-L, Sommerich CM. Biomechanical evaluation of the Eco-Pick lift assist: A device designed to facilitate product selection tasks in distribution centers. Appl Ergon 44: 230236, 2013. doi: 10.1016/j.apergo.2012.07.006.

56. Madinei S, Alemi MM, Kim S, Srinivasan D, Nussbaum MA. Biomechanical assessment of two back-support exoskeletons in symmetric and asymmetric repetitive lifting with moderate postural demands. Appl Ergon 88: 103156, 2020. doi: 10.1016/j.apergo.2020.103156.

57. Madinei S, Alemi MM, Kim S, Srinivasan D, Nussbaum MA. Biomechanical Evaluation of Passive Back-Support Exoskeletons in a Precision Manual Assembly Task: "Expected" Effects on Trunk Muscle Activity, Perceived Exertion, and Task Performance. Hum Factors J Hum Factors 
Ergon Soc 62: 441-457, 2020. doi: 10.1177/0018720819890966.

58. Maher CG. A systematic review of workplace interventions to prevent low back pain. Aust J Physiother 46: 259-269, 2000. doi: 10.1016/S0004-9514(14)60287-7.

59. Marras WS, Lavender SA, Leurgans SE, Rajulu SL, Gary Allread W, Fathallah FA, Ferguson SA. The role of dynamic three-dimensional trunk motion in occupationally-related low back disorders: The effects of workplace factors, trunk position, and trunk motion characteristics on risk of injury. Spine (Phila Pa 1976) 18: 617-628, 1993. doi: 10.1097/00007632-19930400000015.

60. McGill SM, Norman RW, Sharratt MT. The effect of an abdominal belt on trunk muscle activity and intra-abdominal pressure during squat lifts. Ergonomics 33: 147-160, 1990. doi: 10.1080/00140139008927106.

61. McLaughlin JE, King GA, Howley ET, Bassett, Jr DR, Ainsworth BE. Validation of the COSMED K4 b2 Portable Metabolic System. Int J Sports Med 22: 280-284, 2001. doi: 10.1055/s2001-13816.

62. Pandolf KB, Givoni B, Goldman RF. Predicting energy expenditure with loads whiZe standing or walking very slowly. J Appl Physiol 43: 577-581, 1977.

63. Parr BB, Strath SJ, Bassett DR, Howley ET. Validation of the Cosmed K4b2 portable metabolic measurement system. Med Sci Sport Exerc 33: S300, 2001. doi: 10.1097/00005768200105001-01691.

64. Peñailillo L, Blazevich AJ, Nosaka K. Factors contributing to lower metabolic demand of eccentric compared with concentric cycling. J Appl Physiol 123: 884-893, 2017. doi: 10.1152/japplphysiol.00536.2016.

65. Punnett L, Prüss-Ütün A, Nelson DI, Fingerhut MA, Leigh J, Tak S, Phillips S. Estimating the global burden of low back pain attributable to combined occupational exposures. Am J Ind Med 48: 459-469, 2005. doi: 10.1002/ajim.20232.

66. Rahman T, Ramanathan R, Seliktar R, Harwin W. A simple technique to passively gravitybalance articulated mechanisms. J Mech Des Trans ASME 117: 655-657, 1995. doi: $10.1115 / 1.2826738$.

67. Ranavolo A, Varrecchia T, Rinaldi M, Silvetti A, Serrao M, Conforto S, Draicchio F. Mechanical lifting energy consumption in work activities designed by means of the "revised NIOSH lifting equation.” Ind Health 55: 444-454, 2017. doi: 10.2486/indhealth.2017-0075.

68. Ryschon TW, Fowler MD, Wysong RE, Anthony A-R, Balaban RS. Efficiency of human skeletal muscle in vivo: comparison of isometric, concentric, and eccentric muscle action. $J$ Appl Physiol 83: 867-874, 1997. doi: 10.1152/jappl.1997.83.3.867.

69. Sharp MA, Harman E, Vogel JA, Knapik JJ, Legg SJ. Maximal aerobic capacity for repetitive lifting: comparison with three standard exercise testing modes. Eur J Appl Physiol Occup Physiol 57: 753-760, 1988. doi: 10.1007/BF01075999.

70. Shirazi-Adl A. Finite-element evaluation of contact loads on facets of an L2-L3 lumbar segment in complex loads. Spine (Phila Pa 1976) 16: 533-41, 1991.

71. Silverstein B, Clark R. Interventions to reduce work-related musculoskeletal disorders. $J$ Electromyogr Kinesiol 14: 135-152, 2004. doi: 10.1016/j.jelekin.2003.09.023.

72. Simon AA, Alemi MM, Asbeck AT. Kinematic effects of a passive lift assistive exoskeleton. $J$ Biomech 120: 110317, 2021. doi: 10.1016/j.jbiomech.2021.110317.

73. Snook SH. Criteria for Manual Materials Handling-Fact or Fiction. In: 22nd Annual Conference of AIIE. Boston: 1971.

74. Sparto PJ, Parnianpour M, Reinsel TE, Simon S. The Effect of Fatigue on Multijoint Kinematics, Coordination, and Postural Stability During a Repetitive Lifting Test. J Orthop Sport Phys Ther 25: 3-12, 2013. doi: 10.2519/jospt.1997.25.1.3.

75. St-Vincent M, Tellier C, Lortte M. Training in handling: an evaluative study. Ergonomics 32: 191-210, 1989. doi: 10.1080/00140138908966079.

76. Steffens D, Maher CG, Pereira LSM, Stevens ML, Epi MC, Oliveira VC, Chapple M, 
Teixeira-salmela LF, Hancock MJ. Prevention of Low Back Pain. JAMA Intern Med 2000: 110, 2016. doi: 10.1001/jamainternmed.2015.7431.

77. SuitX. SuitX BackX. 2017.

78. Ulrey BL, Fathallah FA. Effect of a personal weight transfer device on muscle activities and joint flexions in the stooped posture. J Electromyogr Kinesiol 23: 195-205, 2013. doi:

10.1016/j.jelekin.2012.08.014.

79. Watanabe M, Kaneoka K, Okubo Y, Shiina I, Tatsumura M, Miyakawa S. Trunk muscle activity while lifting objects of unexpected weight. Physiotherapy 99: 78-83, 2013. doi: 10.1016/j.physio.2011.09.005.

80. Wehner M, Rempel D, Kazerooni H. Lower Extremity Exoskeleton Reduces Back Forces in Lifting. ASME 2009 Dyn Syst Control Conf Vol 2 : 49-56, 2009. doi: 10.1115/DSCC2009-2644.

81. Welbergen E, Kemper HCG, Knibbe JJ, Toussaint HM, Clysen L. Efficiency and effectiveness of stoop and squat lifting at different frequencies. Ergonomics 34: 613-624, 1991. doi: 10.1080/00140139108967340.

82. Whitfield BH, Costigan PA, Stevenson JM, Smallman CL. Effect of an on-body ergonomic aid on oxygen consumption during a repetitive lifting task. Int J Ind Ergon 44: 39-44, 2014. doi: 10.1016/j.ergon.2013.10.002.

83. Yang C-J, Zhang J-F, Chen Y, Dong Y-M, Zhang Y. A Review of exoskeleton-type systems and their key technologies. Proc Inst Mech Eng Part C J Mech Eng Sci 222: 1599-1612, 2008. doi: 10.1243/09544062JMES936.

84. Yang H, Haldeman S, Lu M-L, Baker D. Low Back Pain Prevalence and Related Workplace Psychosocial Risk Factors: A Study Using Data From the 2010 National Health Interview Survey. J Manipulative Physiol Ther 39: 459-472, 2016. doi: 10.1016/j.jmpt.2016.07.004. 